\title{
Tendon-Sheath Mechanisms in Flexible Membrane Wing Mini-UAVs: Control and Performance
}

\author{
Tegoeh Tjahjowidodo and Shian Lee \\ School of Mechanical and Aerospace Engineering, Nanyang Technological University, 50 Nanyang Avenue, \\ North Spine (N3), Singapore 639798 \\ Correspondence should be addressed to Tegoeh Tjahjowidodo; ttegoeh@ntu.edu.sg \\ Received 9 February 2017; Accepted 1 June 2017; Published 24 July 2017 \\ Academic Editor: Mahmut Reyhanoglu
}

Copyright (C) 2017 Tegoeh Tjahjowidodo and Shian Lee. This is an open access article distributed under the Creative Commons Attribution License, which permits unrestricted use, distribution, and reproduction in any medium, provided the original work is properly cited.

\begin{abstract}
Flexible membrane wings (FMWs) are known for two inherent advantages, that is, adaptability to gusty airflow as the wings can flex according to the gust load to reduce the effective angle of attack and the ability to be folded for compact storage purposes. However, the maneuverability of UAV with FMWs is rather limited as it is impossible to install conventional ailerons. The maneuver relies only on the rudders. Some applications utilize torque rods to warp the wings, but this approach makes the FMW become unfoldable. In this research, we proposed the application of a tendon-sheath mechanism to manipulate the wing shape of UAV. Tendon-sheath mechanism is relatively flexible; thus, it can also be folded together with the wings. However, its severe nonlinearity in its dynamics makes the wing warping difficult to control. To compensate for the nonlinearity, a dedicated adaptive controller is designed and implemented. The proposed approach is validated experimentally in a wind tunnel facility with imitated gusty condition and subsequently tested in a real flight condition. The results demonstrate a stable and robust wing warping actuation, while the adaptive washout capability is also validated. Accurate wing warping is achieved and the UAV is easily controlled in a real flight test.
\end{abstract}

\section{Introduction}

Recently mini-UAVs have been a very important vehicle class in various tasks. Being extremely stealthy and also cheaper and safer to operate than a manned aircraft, a mini-UAV is a suitable aircraft for flying into dangerous zones such as enemy territory for scouting missions. They can be used for border patrol missions, which can be mundane and also costly for human pilots. However, conventional mini-UAVs possess a few critical flaws which hinder the progress of their usage in the aforementioned missions. Due to the size of a miniUAV being typically $1 \mathrm{~m}$ or more in wingspan, a conventional rigid wing mini-UAV is usually disassembled for storage and also transportation to the mission site. Thereafter, the human operator has to reassemble the mini-UAV at the site, while being exposed to the elements. In addition, the time required to assemble the mini-UAV can be a critical disadvantage to a surveillance mission as the target may have left the site. A mini-UAV is also quite difficult to pilot in gusty environments, especially in the climates near the equator with frequent thunderstorms [1]. Due to the very small moment of inertia, a mini-UAV can be a challenge to control in windy conditions. Currently, autopilots with a closed-loop feedback coupled with responsive actuators are being used to stabilize and reject the gusts [2]. This consumes the limited energy on board of a mini-UAV, which is very valuable in order to stay airborne for as long as possible. Actuator saturation may also occur, which lead to a mini-UAV not being able to react quickly enough to reject the gust response.

Flexible membrane wing (FMW) mini-UAVs possess very interesting properties such as adaptive washout [3]; also an FMW can be folded spanwise towards the fuselage for storage and quick deployment purposes. These are huge advantages over the conventional rigid wing. An FMW miniUAV can be launched immediately by the operator, without any disassembly or assembly process. The operator can also be shielded from the dangerous elements by launching an FMW mini-UAV from inside a vehicle, as illustrated by Prioria 


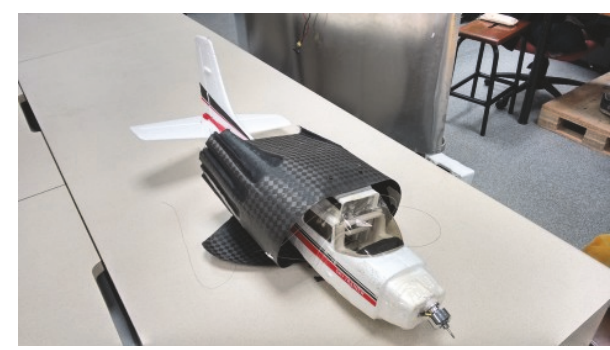

(a)

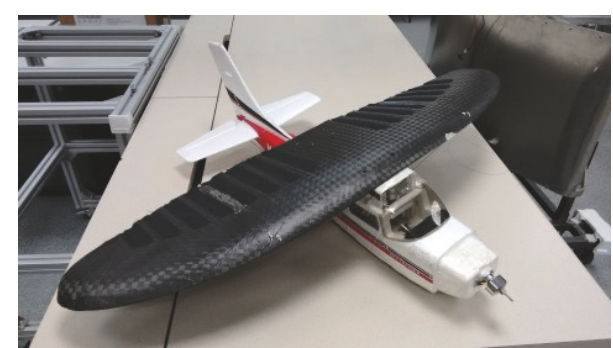

(b)

FIGURE 1: The FMW in the folded and unfolded states: (a) flexible membrane wing folded around the fuselage of the mini-UAV; (b) flexible membrane wing deployed.

Robotics Maveric UAS. Adaptive washout, which is another desired feature, can dampen the longitudinal gusts response by flexing the airfoil away from the direction of the gusts, thereby decreasing the effects of gusts on an FMW. The ability to flex the wings in the chordwise direction allows an FMW to handle a higher angle of attack $(\alpha)$, therefore delaying the stall response.

However, due to the very thin airfoil, an FMW is unable to house an aileron as the material of the FMW is made of nylon cloth and carbon fiber strips to support the nylon cloth. Previous research has suggested other techniques such as utilizing torque rods and Kevlar lines to warp an FMW $[4,5]$. Even though the warping ensues and also the roll rate is satisfactory, the biggest drawback of these systems is that they hinder the downward folding ability of an FMW. A method of warping the wings to provide sufficient roll rate which also does not hinder the two main properties of an FMW (downwards folding and adaptive washout) is required.

In this paper, a novel method that utilizes the tendonsheath mechanism (TSM) is discussed to fulfill the requirement mentioned above. However, a TSM is not without its own disadvantage, as it experiences severe nonlinear behavior during the actuation. Nevertheless, it has been shown that the TSM nonlinearity behaviors can be modeled and controlled [6]. Similar procedure is carried out in the case of the FMW warping via TSM; the nonlinear hysteresis is identified, modeled, and, subsequently, compensated with a proper controller strategy.

This paper is structured as follows. In Section 2, a brief and concise introduction to the attributes and characteristics of the FMW is provided. The fabrication procedures of the FMW are also illustrated as well. Section 3 includes the design details of the FMW mini-UAV. The characterization of the mini-UAV is also discussed. Section 4 provides some crucial information about the TSM, the nonlinear behaviors, and the procedures carried out to model such behaviors. Section 5 provides details about the control structure implemented in the FMW warping via TSM. Extensive wind tunnel tests using both steady and gusty winds were carried out for the FMW mini-UAV, and the results and discussions are showed in Section 6. In Section 7, the flight test results are presented and discussed. Finally, a conclusion is drawn in Section 8.
TABLE 1: Specifications of the FMW.

\begin{tabular}{lc}
\hline Wingspan & $1 \mathrm{~m}$ \\
Chord length & $0.25 \mathrm{~m}$ \\
Mean aerodynamic chord & $0.22 \mathrm{~m}$ \\
Area & $0.19 \mathrm{~m}^{2}$ \\
Aspect ratio & 5.158 \\
Weight & $160 \mathrm{~g}$ \\
\hline
\end{tabular}

\section{Flexible Membrane Wing}

Introduced by [3], the FMW is a piece of thin airfoil wing, similar to the wing of a bat. The ribs of the FMW are made of carbon fiber reinforced plastic (CFRP), while the skin of the FMW is made of nylon cloth. Most of the aerodynamic forces are carried by the nylon cloth, and the cloth is supported and stretched across the CFRP ribs. The detailed specification of the FMW investigated in this paper is given in Table 1. The FMW is able to withstand up to $35 \mathrm{~N}$ of lift without buckling when tested in a wind tunnel; therefore, it is considered to be very light for its strength and size.

Many different rib configurations have been tested and an optimized configuration has been identified [3]. Other similar FMWs available in $[4,5]$ were also created in a similar configuration, with the ribs attached to the main spar at the leading edge of the FMWs. The aerodynamics of the FMW has been studied in detail in [7], and the optimization of the shape of the FMW has been investigated in [8]. The effects of the ratio of ribs and fabric have been investigated thoroughly in [9], and an optimized ratio has been found, which generates the maximum lift over drag performance. The fabricated FMW used in this research, therefore, follows the guideline presented by $\mathrm{Hu}$ et al. [9].

2.1. Characteristics and Features. The main feature of the FMW is the downwards folding ability for storage and quick launching purposes (Figure 1 illustrates a typical FMW miniUAV on storage and unfolding states). Due to its flexible nature in the spanwise direction, coupled with a unique thin airfoil with camber, the FMW is able to only fold downwards easily while resisting aerodynamic load that tries to curl the wing upwards. Experiments in a wind tunnel showed that the 
FMW is able to withstand up to $35 \mathrm{~N}$ of lift force without buckling upwards. However, only very little force is needed to bend the wing downwards and to fold around the fuselage; hence, the FMW mini-UAV is not suitable in a negative flight condition. More discussions about the wind tunnel experiments will be presented in Section 6 .

The chordwise flexibility of the FMW provides the ability to dampen the longitudinal gusts, also known as adaptive washout. This passive phenomenon allows the FMW to adapt to gusty conditions during flight, despite a stable limit cycle oscillation that might appear on the FMW structure [3]. The FMW is able to dampen the gusts, that is, by flexing in the chordwise direction and by changing the effective and airfoil shape. When a gust load attacks from lower side of the wing (e.g., a case of high $\alpha$ ), in typical conventional rigid wings, a sudden increase of lift forces will occur. However, for the FMW, the wing will adapt its shape and decrease the lift coefficient $\left(C_{L}\right)$, but the total amount of lift experienced by the FMW will not be perturbed by much. Once the gust is over, the FMW will spring back to its original shape, recovering $C_{L}$. Same case applies when there is a perturbation in the headwind. When the airspeed increases or decreases, the FMW will be adapted accordingly to provide a near constant lift force. Tuning is required to have a functional adaptive washout mechanism and the FMW used in this paper has been constructed after numerous times of experimentation to achieve the desired result.

Flexibility in the chordwise direction of the FMW has taken advantage for wing warping. In $[5,11]$, the FMW was installed with torque rods to induce wing warping. Sufficient roll rates were achieved and the torque rods could induce an almost pure rolling moment without yawing moment. However, the major drawback is that the FMW cannot be folded downwards, as the torque rods are stiff and rigid structures. Kevlar cables have been tried as well [11]; however, the tensioned Kevlar cables produced more spanwise warping (wing curling) than chordwise; therefore, this mechanism is unable to provide sufficient roll rates. In addition, the exposed cables are also prone to breaking during landings and will be tangled easily if the FMW is folded. Wing warping is a very delicate mechanism which relies on an exact amount of flexibility and stiffness. To facilitate wing warping, the structural stiffness of the FMW must be optimized, as the wing has to be flexible enough to allow deformations and yet stiff enough to withstand the aerodynamic loads. Conflicting conditions are presented when choosing the stiffness of the FMW. If a high stiffness is chosen for the FMW, huge amounts of energy will be required to warp the structure. Yet, if a low stiffness is chosen, the wing will not be able to bear the aerodynamic loads [12]. A few iterations have to be carried out to obtain the stiffness value that can compromise the need for warping, folding, and aerodynamic loads. The optimal stiffness is characterized by folding properties and aerodynamics properties that will be discussed in Sections 4 and 6.

2.2. Fabrication Procedures. The fabrication of the carbon fiber membrane wing involves successive steps with different materials, equipment, and handling techniques. First of all, a

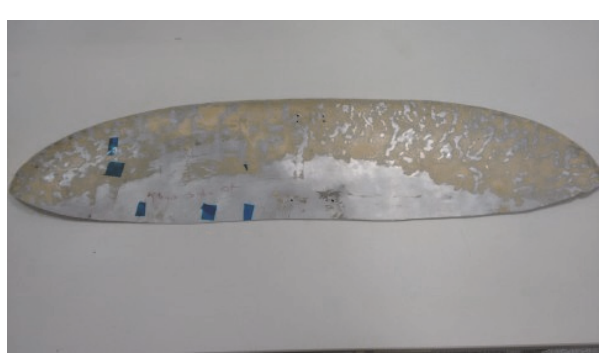

FIGURE 2: 3D aluminum mold.

female mold made of aluminum (Figure 2) is designed and then fabricated. Aluminum is chosen as the material for the mold, as it can withstand the pressure and also the heat of the autoclave curing process.

A template board is used to guide the cutting of the carbon fiber prepreg. After cutting 3 copies of carbon fiber wing platform, they will be layered on top of each other forming the leading edge and main structure of the wings. Unidirectional carbon fiber strips are cut and then placed on the main carbon fiber wing platform. Six layers of carbon fiber reinforcement strips are used in this fabrication process to provide a suitable stiffness. The nylon cloth is then layered together with the carbon fiber to form the FMW, precured. The whole assembly will be placed on top of the aluminum mold and then taped on to be kept in place. A vacuum bag will be placed around the assembly, and a bag sealant tape is used to secure the vacuum bag with the aluminum mold to ensure an airtight condition. The carbon fiber layup is cured under high temperature and pressure in the autoclave, using the built in program "Autoclave 10743." After the curing cycle is complete, the carbon fiber wing will be separated from the mold and the vacuum bag. Extra bits of the wing are then trimmed off and the FMW fabrication is complete. Although the fabrication process is repeatable, however, the final product depends on the operator's skill; that is, layering the carbon fiber and the nylon cloth, enveloping with a vacuum bag, vacuuming, and demolding (release the parts from the mould) require some experiences.

\section{Flexible Membrane Wing Mini-UAV}

A mini-UAV was designed and built in order to validate the TSM actuated FMW warping. The design process of the UAV was done by identifying the high-level requirements, for example, payload weight and size, flight endurance, cruising speed, and top speed; and subsequently, the steps described in $[13,14]$ were followed to guide the aircraft sizing and geometry decisions.

3.1. Design and Build. As the wing is required to fold around the fuselage for storage, the FMW is installed at the top of the fuselage, with a top wing configuration. The payload of the mini-UAV is a visual camera to record the flight. Various electronics, for example, servos, autopilot microcontroller board, and electronic speed controller, are also required, not to mention the source of energy which will be a LithiumPolymer battery. The location of the electronics and battery 


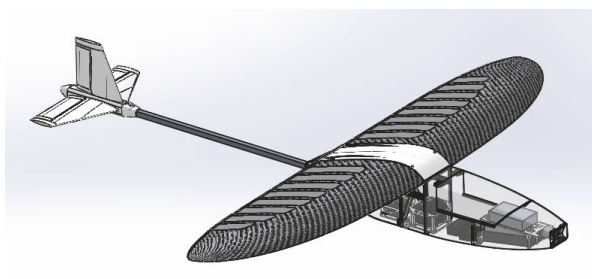

(a)

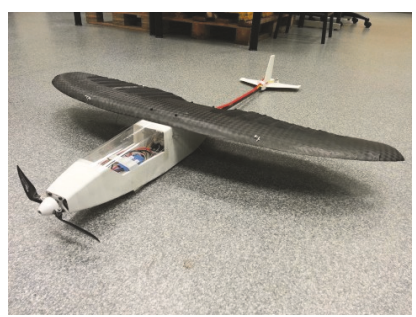

(b)

FIGURE 3: Design of the FMW mini-UAV and the final prototype: (a) 3D CAD model of the FMW mini-UAV; (b) 3D printed fuselage of the FMW mini-UAV. Carbon fiber rod is used for the tail boom.

are critical as they will affect the center of gravity of the aircraft. The total weight of the aircraft is limited to $1.2 \mathrm{~kg}$ in order to fulfill the stall speed, take-off speed, and cruising speed requirements. Careful decisions on the locations of the electronics and battery are made to respect the center of gravity. The $3 \mathrm{~d}$ CAD model of the mini-UAV is shown in Figure 3(a), and the fabricated FMW mini-UAV is illustrated in Figure 3(b).

The preliminary sizing of the aircraft was done to house all the electronics and payload inside the fuselage. After designing the fuselage, the tail design was done by limiting the horizontal tail span to $0.3 \mathrm{~m}$, for the tube launch capability in the future. The horizontal tail aspect ratio $\mathrm{AR}_{\mathrm{HT}}$ was recommended to be $2 / 3$ of the wing aspect ratio [15]; therefore,

$$
\begin{aligned}
\mathrm{AR}_{\mathrm{HT}} & =\frac{2}{3} \mathrm{AR}_{W}, \\
S_{\mathrm{HT}} & =\frac{b_{\mathrm{HT}}^{2}}{\mathrm{AR}_{\mathrm{HT}}}=0.0262 \mathrm{~m}^{2},
\end{aligned}
$$

where $\mathrm{AR}_{W}$ is the wing aspect ratio, $b_{\mathrm{HT}}$ is the horizontal tail span, and $S_{\mathrm{HT}}$ is the horizontal tail area. The tail volume ratio $C_{\mathrm{HT}}$ was chosen to be 0.5 , as normally used in typical miniUAVs [13-15]. Therefore, the horizontal tail moment arm, $l_{\mathrm{HT}}$, can be found with the tail volume ratio equation:

$$
\begin{aligned}
C_{\mathrm{HT}} & =\frac{l_{\mathrm{HT}} S_{\mathrm{HT}}}{C^{\prime}{ }_{W} S_{W}}, \\
l_{\mathrm{HT}} & =0.806 l,
\end{aligned}
$$

where $C^{\prime}{ }_{W}$ is the mean aerodynamic chord and $S_{W}$ is the wing surface area.

3.2. UAV Characterization via Empirical Methods. A few characteristics of the FMW mini-UAV are difficult to be obtained mathematically; therefore empirical tests are performed; namely, the aerodynamic data, natural frequencies, and moment of inertia of the FMW mini-UAV are obtained experimentally through wind tunnel testing (shown in Section 6). Modal testing was performed through an impact hammer testing to study the natural frequencies of the FMW, and finally, the actual moment of inertia of the entire miniUAV structure was identified using the bifilar pendulum method.
The fundamental frequency of the FMW is particularly important as the flexibility of the wing will induce fluttering if the gust frequency happens to be the natural frequency of the FMW. For the FMW, the fundamental frequency was determined experimentally via modal testing. In particular, the first mode is the most critical to the FMW, as the deflection will be the greatest. From Figure 4(a), it can be observed that the first mode of the FMW occurs around $7 \mathrm{~Hz}$. The coherence diagram (Figure 4(b)) shows a peak at $7 \mathrm{~Hz}$, signifying high confidence of accuracy on the natural frequency. A slow motion video was captured on the vibration of the FMW, and the vibration frequency of the FMW from the video was shown to be $7 \mathrm{~Hz}$ as well.

A bifilar vertical-axis torsional pendulum test was performed to find out the moment of inertia of the mini-UAV in the longitudinal direction. Strings were attached to the center of gravity of the UAV, and then the UAV was hung from the ceiling as a bifilar pendulum. The following equation shows the moment of inertia of the mini-UAV:

$$
I=\frac{m g T^{2} l_{p}^{2}}{16 \pi^{2} L}=0.016975 \mathrm{kgm}^{2},
$$

where $m$ is the mass of the mini-UAV, $g$ is the gravitational acceleration, $T$ is the rotational oscillation period of the bifilar pendulum, $l_{p}$ is the length of the bifilar pendulum, and $L$ is the length of the supporting strings for the bifilar pendulum. The moment of inertia is of interest because the roll rate of the mini-UAV given a rolling moment can be found if the moment of inertia is known.

\section{Tendon-Sheath Mechanism}

Inspired by the tendon-sheath tissue found inside human hands, engineers have been applying TSM to construct, for example, power transmission systems in robotic hands and fingers, as it can be routed in a very flexible way [16]. The TSM is comprised of a piece of tendon, which slides along inside the sheath covering the tendon. The tendon is made of Teflon coated metal wire, and the sheath is made of a metal coil, much like a spring around the tendon. This helps to reduce the friction between the tendon and the sheath.

4.1. Force Transmission. Only tensile forces can be transmitted via the TSM. When tension is applied at the input side of 


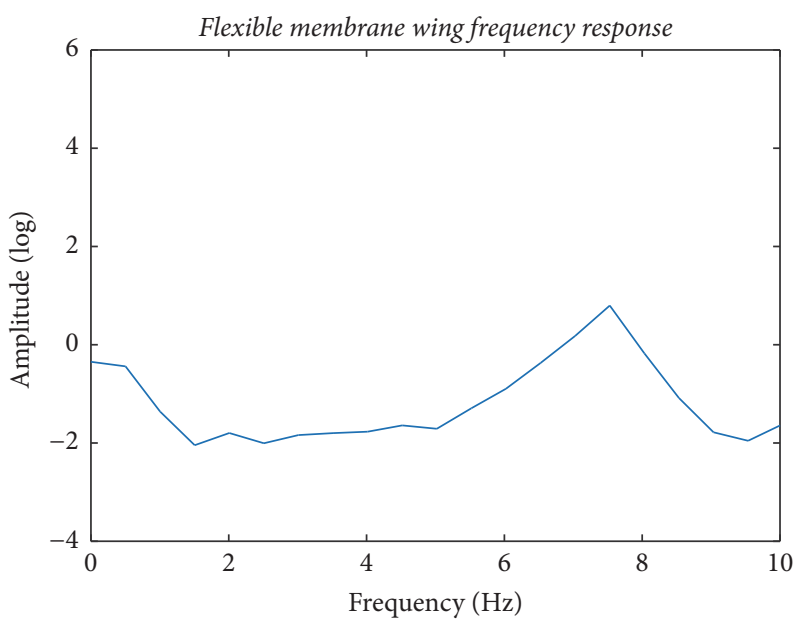

(a)

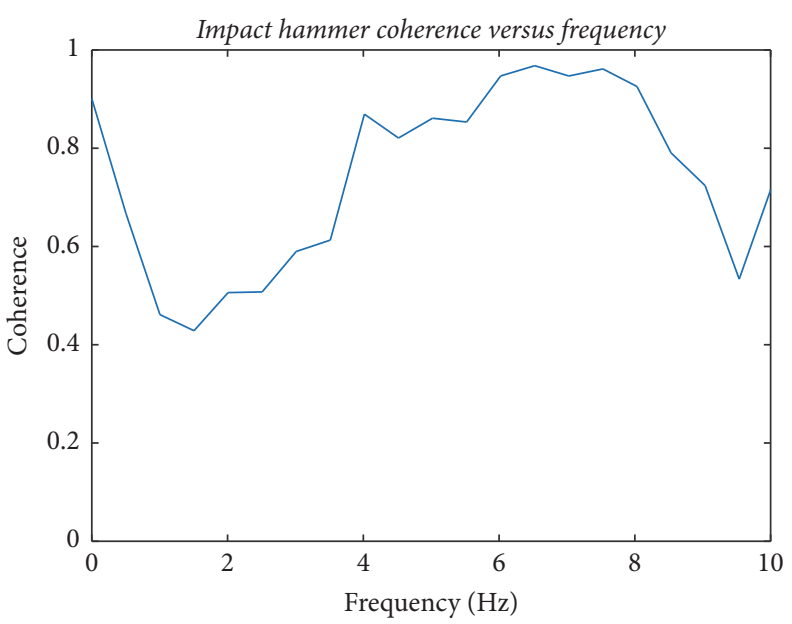

(b)

FIGURE 4: Results of the modal testing of the FMW: (a) frequency response of the FMW obtained from modal testing; (b) coherence of the frequency response.

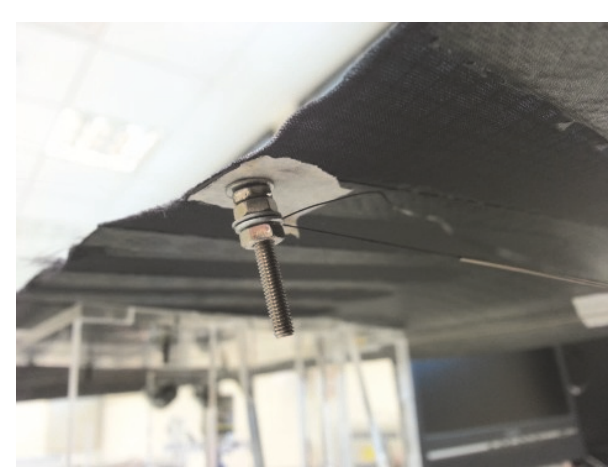

FIgURE 5: The trailing edge of the FMW is fitted with the tendon.

the TSM, the tendon becomes tighter and therefore starts to slide along the sheath, creating a pulling force at the output side of the TSM. The relationship between the tension input and the output is not linear; therefore, a controller is needed when accurate positioning control is required [16]. Typically, a high pretension is given to the tendon, in order to increase the efficiency and to achieve a quicker response. TSM is useful for FMW warping because the tendon-sheath is flexible and is possible to mount it without requiring much space. The trailing edge of the wing is connected to the output side of the TSM (Figure 5), and the servomotor is connected to the input side of the TSM. Therefore, tensile forces can be transmitted from the servomotor placed inside the fuselage to the trailing edge of the FMW in order to control the wing warping. The flexibility of the TSM allows the FMW to fold downwards for storage purposes, which is the primary objective.

4.2. Nonlinear Behaviors. Although the tensile forces can be transmitted via the TSM to induce wing warping, the inputoutput relationship of the tensile forces is not linear. The nonlinear behavior, which is caused by the friction and also backlash of the TSM, can cause severe inaccuracy in adjusting

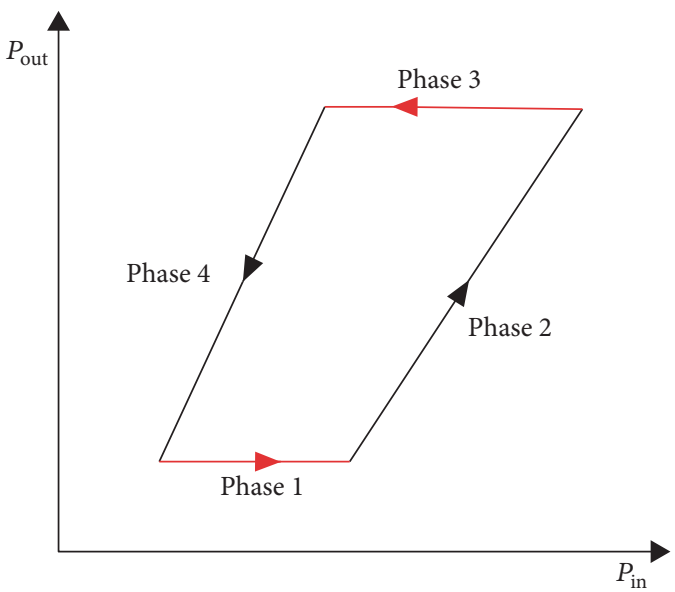

FIGURE 6: Relation between position input and position output [10].

the wing warping if no appropriate controller is applied. Nonetheless, the nonlinear behaviors can be modeled and controlled, as shown in various works [6].

In [6], the hysteresis behavior of the FMW warping via TSM was modeled using the Generalized Bouc-Wen model. The Generalized Bouc-Wen model from [17] is suitable for the FMW warping via TSM because of the asymmetric properties of the hysteresis curves. Typically, the hysteresis behavior of the TSM is categorized in 4 different phases, as illustrated in Figure 6. Phases 1 and 2 occur when tensile forces are applied from the input side, while Phases 3 and 4 occur when the tensile forces are stopped, and the mechanism is springing back into the original position. For a typical TSM, the hysteresis diagram shows sharp and distinct changes between the phases [10]. However, it was found that the hysteresis behaviors of the FMW warping via TSM were not the same, as the phase change occurs gradually, showing a gradual change in slopes between Phases 1 and 2, as well as in between Phases 3 and 4 . This phenomenon has been 


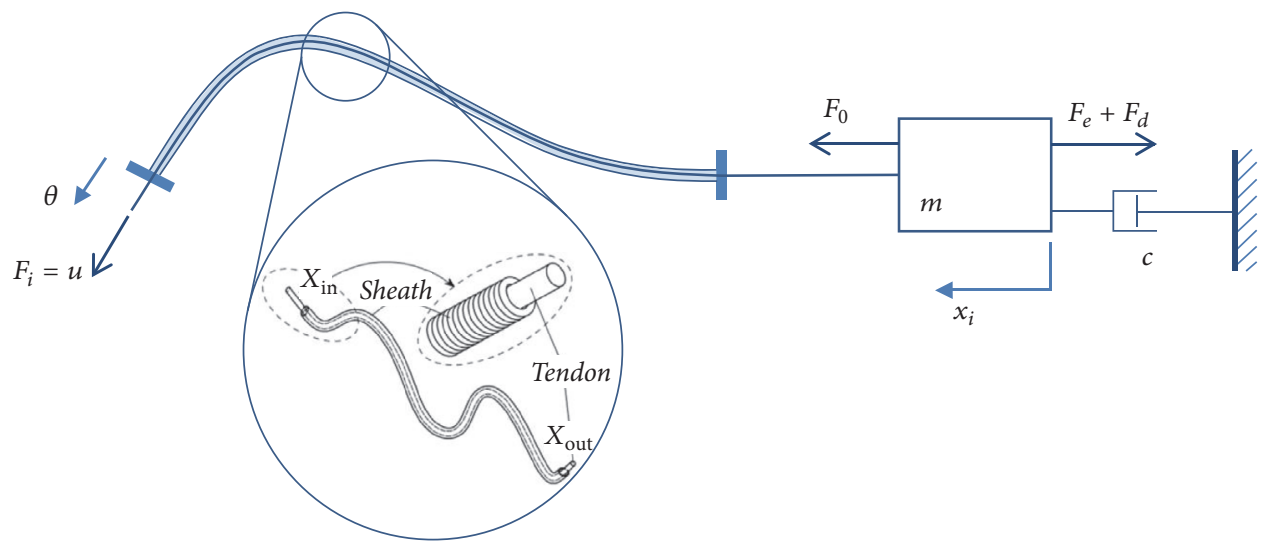

FIGURE 7: Force transmission on a TSM.

investigated in [10] and has been found to be the result of a low pretension in the tendon.

\section{Adaptive Backstepping Controller}

As the FMW warping via TSM is a highly nonlinear system [6], an appropriate control strategy is necessary to help in controlling the position of the wing warping more accurately. The simplest way to deal with systems with hysteresis nonlinear behavior is by implementing the model in a feedforward loop, combined with a feedback loop to compensate for some disturbances and uncertainties (see, e.g., [10]). In this paper, an adaptive backstepping controller is proposed and the results are benchmarked to the performance of feedforward/feedback controller in [10].

The dynamic friction force, $F$, for the TSM can be described as follows [18]:

$$
\begin{aligned}
& F=k_{x} \phi x_{i}+k_{\zeta} \zeta+v \dot{x}_{i}+F_{\Theta}, \\
& \dot{\zeta}=\rho\left(\dot{x}_{i}-\sigma\left|\dot{x}_{i}\right||\zeta|^{n-1} \zeta+(\sigma-1) \dot{x}_{i}|\zeta|^{n}\right), \\
& \phi=\frac{e^{2 \dot{x}_{i}}+\tanh \left(x_{i}\right) \tanh \left(\ddot{x}_{i}\right)}{e^{2 \dot{x}_{i}}+1}
\end{aligned}
$$

where $x_{i} ; \dot{x}_{i} ; \ddot{x}_{i}$ are the position, velocity, and acceleration at the actuator side, respectively; $F$ is the total friction forces at the TSM; $k_{\zeta}$ is a factor that expresses the ratio of the internal state, $\zeta$, to the friction force; $k_{x}$ is the stiffness factor that controls separate curves of the hysteresis loops; $\rho>0$; $\sigma>0.5 ; n \geq 1$ are coefficients that control the shape and size of hysteresis loops for the friction force; $\tanh (\cdot)$ is the hyperbolic tangent function which is defined as $\tanh (\cdot)=$ $\left(e^{2(\cdot)}-1\right) /\left(e^{2(\cdot)}+1\right) ; v$ is the viscous coefficient; and $F_{\Theta}$ is a offset point of the friction force; the dot at the top of variables represents the first derivative with respect to time.

Remark 1 . It is noted that the variable $\zeta$ given by (4)-(6) is uniformly bounded for any piecewise continuous signals $x_{i}$; $\dot{x}_{i}$ (see $[19,20]$ for more details).
The dynamic model for the TSM-driven side with the mass, $m$, damping coefficient, $c$, input torque, $F_{o}$, external disturbance torque, $F_{d}$, with unknown bound, and environmental torque, $F_{e}$, as shown in Figure 7 can be described as [21]

$$
m \ddot{\theta}+c \dot{\theta}=F_{o}-F_{e}-F_{d}
$$

where $\theta$ is the angular displacement of the output that corresponds to the wing warp.

The relation between the input force $F_{i}=u$, where $u$ is the control input for the system, and the output force $F_{o}$ can be expressed as $F_{i}=F_{o}+F$ where $F$ is the total friction forces between the tendons and the sheaths. Equation (7) can be rewritten by

$$
m \ddot{\theta}+c \dot{\theta}=(u-F)-F_{e}-F_{d}
$$

We define the system states as $x=\left[x_{1} ; x_{2}\right]^{T}=[\theta ; \dot{\theta}]^{T}$; then the dynamics of the joint in the tendon-sheath system can be simplified as

$$
\begin{aligned}
\dot{x}_{1} & =x_{2}, \\
\dot{x}_{2} & =\frac{1}{m}\left\{(u-F)-F_{e}-F_{d}-c x_{2}\right\}, \\
\theta & =x_{1},
\end{aligned}
$$

where the friction force $F$ is described by (4). Then the variable $\dot{x}_{2}$ given in (10) can be rewritten as

$$
\begin{aligned}
\dot{x}_{2} & =\frac{1}{m}\left\{\left(u-k_{x} \phi x_{i}-v \dot{x}_{i}\right)-c x_{2}+D\right\} \\
& =\frac{1}{m}(u+D)+\Theta^{T} \varphi
\end{aligned}
$$


where $\varphi ; D ; \Theta$ are expressed with the following forms:

$$
\begin{aligned}
\varphi & =\left[\phi x_{i}, \dot{x}_{i}, x_{2}\right]^{T} \\
& =\left[x_{i}\left\{e^{2 \dot{x}_{i}}+\frac{\tanh \left(x_{i}\right) \tanh \left(\ddot{x}_{i}\right)}{e^{2 \dot{x}_{i}}+1}\right\}, \dot{x}_{i}, x_{2}\right]^{T}, \\
D & =-\left(k_{\zeta} \zeta+F_{o}\right)-F_{e}-F_{d}, \\
\Theta & =\left[-\frac{k_{x}}{m},-\frac{v}{m},-\frac{c}{m}\right]^{T} .
\end{aligned}
$$

To design the controller $u$ and suitable adaptive laws, some assumptions are needed: (i) the output and input positions, $\theta$ and $x_{i}$, are measurable; (ii) the desired trajectories $\theta_{r}, \dot{\theta}_{r}, \ddot{\theta}_{r}$ are continuous and bounded; (iii) the environmental torque $F_{e}$ and external disturbance $F_{d}$ are bounded by unknown bounds. From Remark 1 and assumption (iii), the variable $D$ is bounded by an unknown variable $D^{*}(|D| \leq$ $D^{*}$ ) because $\zeta ; F_{e}, F_{d}$ are bounded.

To use the backstepping technique $[22,23]$, change of coordinate is made to system (9)-(12):

$$
\begin{aligned}
& \xi_{1}=x_{1}-\theta_{r} \\
& \xi_{2}=x_{2}-\dot{\theta}_{r}+\alpha_{v 1} \xi_{1},
\end{aligned}
$$

where $\alpha_{v 1}$ is a positive designed parameter that will be determined later.

From (14) and (15), the new coordinates for the system in (9)-(12) can be expressed by

$$
\begin{aligned}
& \dot{\xi}_{1}=\xi_{2}-\alpha_{v 1} \xi_{1}, \\
& \dot{\xi}_{2}=\frac{1}{m}(u+D)+\Theta^{T} \varphi-\ddot{\theta}_{r}+\alpha_{v 1} \dot{\xi}_{1} .
\end{aligned}
$$

Let $\widehat{D}^{*}, \widehat{m}, \widehat{\Theta}$, and $\widetilde{D}^{*}=D^{*}-\widehat{D}^{*}, \widetilde{m}=m-\widehat{m}$, and $\widetilde{\Theta}=\Theta-\widehat{\Theta}$ be the estimates and error estimates of $D^{*}, m$, and $\Theta$, respectively. Define a virtual control input, $\bar{u}$, such that $u=\widehat{m} \bar{u}-\widehat{D}^{*} \tanh \left(\xi_{2} / \varepsilon\right)$, where $\varepsilon>0$ will be determined later. From (9)-(12), parameter update laws, $\widehat{D}^{*}, \widehat{m}, \widehat{\Theta}$, and control input, $u$, are designed as follows:

$$
\begin{aligned}
u & =\widehat{m} \bar{u}-\widehat{D}^{*} \tanh \left(\frac{\xi_{2}}{\varepsilon}\right), \\
\bar{u} & =\ddot{\theta}_{r}-\alpha_{v 1} \dot{\xi}_{1}-\xi_{1}-\alpha_{v 2} \xi_{2}-\widehat{\Theta}^{T} \varphi, \\
\dot{\widehat{\Theta}} & =k_{\Theta} \xi_{2} \varphi-\sigma_{1} \widehat{\Theta} \\
\dot{\widehat{m}} & =-k_{m} \xi_{2} \bar{u}-\sigma_{2} \widehat{m}, \\
\dot{\widehat{D}}^{*} & =k_{D} \xi_{2} \tanh \left(\frac{\xi_{2}}{\varepsilon}\right)-\sigma_{3} \widehat{D}^{*},
\end{aligned}
$$

where $\alpha_{v 1} ; \alpha_{v 2} ; k_{\Theta} ; k_{m} ; k_{D} ; \sigma_{i}(i=1 ; 2 ; 3)$ are positive parameters that adjust the designed controller $u$ to force the tracking error, $e_{r}=\theta_{r}-\theta$, to approach the desired values.

With (17) to (21), the following theorem holds.
Theorem 2. Consider the nonlinear system (16) with the designed controller, $u$, from (17) to (18) and adaptive laws (19) to (21); the following statements hold:

(1) The tracking error, $e_{r}$, and adaptive laws, $\widehat{D}^{*}, \widehat{m}, \widehat{\Theta}$, are globally uniformly bounded.

(2) In the presence of unknown model parameters and their bounds, the position tracking error converges to a desired compact region $\Omega=\left\{\left|e_{r}\right||| e_{r} \mid \leq 2 \sqrt{\rho / \Psi}\right\}$,

where

$$
\begin{aligned}
\Psi= & \frac{\sigma_{1}}{2 k_{\Theta}} \Theta^{T} \Theta+0.2785 \varepsilon \frac{D^{*}}{m}+\frac{\sigma_{2}}{2 k_{m}} m \\
& +\frac{\sigma_{3}}{2 m k_{D}}\left(D^{*}\right)^{2}, \\
\rho= & \max \left\{2 \alpha_{v 1}, 2 \alpha_{v 2}, \sigma_{1}, \sigma_{2}, \sigma_{3}\right\} .
\end{aligned}
$$

(See Appendix for the proof.)

Remark 3. For validating the proposed controller and adaptive laws given by (17) to (21), a few simulations have been carried out. Some guidelines are recommended to determine the designed control parameters:

(i) The parameters $\alpha_{v 1}$ and $\alpha_{v 2}$ determine the level of the convergence speed of $e_{r}$. Large values of $\alpha_{v 1}$ and $\alpha_{v 2}$ will lead to faster convergence of the tracking error. However, if these values are too large, it may cause chattering in the controller. High values of parameters $k_{\Theta} ; k_{m} ; k_{D}$ will improve the parameter adaptation speed and tracking performances. In addition, $\varepsilon$ is used to guarantee the smoothness and avoid the discontinuity of the controller $u$ given by (17). A small value of $\varepsilon$ is desired. Therefore, we suggest to keep $\alpha_{v 1}$ and $\alpha_{v 2}$ fixed at acceptable large values and $\varepsilon$ at a small value and then increase the other parameters such as $k_{\Theta} ; k_{m} ; k_{D}$ until the desired tracking performances are achieved.

(ii) The parameters $\sigma_{1} ; \sigma_{2} ; \sigma_{3}$ are used to prevent the estimate values of $\widehat{D}^{*}, \widehat{m}, \widehat{\Theta}$ to be too large. However, large values of $\sigma_{1} ; \sigma_{2} ; \sigma_{3}$ may suppress the prevention. Hence, small values for $\sigma_{1} ; \sigma_{2} ; \sigma_{3}$ are chosen.

To illustrate the proposed controller performance, a dynamic simulation with realistic dynamic friction model parameters are chosen from Do et al. [18], where $k_{x}=$ 0.01083; $\rho=54.658 ; n=2.0458 ; \sigma=1.58 ; k_{\zeta}=0.14368 ; v=$ $0.02686 ; F_{O}=0.0099$. The parameters for the dynamic joint are selected as $m=0.0349 ; c=0.0105$. The environment is simulated as an elastic spring $F_{e}=k_{e} y$, where $k_{e}=0.4185$ and $y$ is the output position. The disturbance is chosen, where $F_{d}=0.2 \sin (0.2 \pi t)$. The designed controller and updated law parameters are selected based on the guidelines given by the Remark 3. With this idea, we choose the control parameters as $\alpha_{v 1}=10 ; \alpha_{v 2}=15 ; k_{\Theta}=0.5 ; k_{m}=0.5 ; k_{D}=1 ; \sigma_{i}=0.01(i$ $=1 ; 2 ; 3) ; \varepsilon=0.05$. The initial condition for updated estimate parameters are chosen such that $\widehat{\Theta}(0)=[0,0,0]^{T} ; \widehat{m}(0)=0$; $\widehat{D}^{*}(0)=0$. 


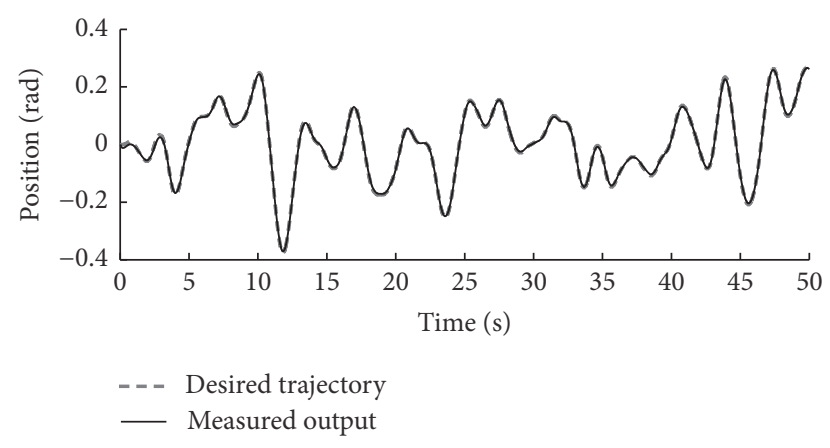

(a)

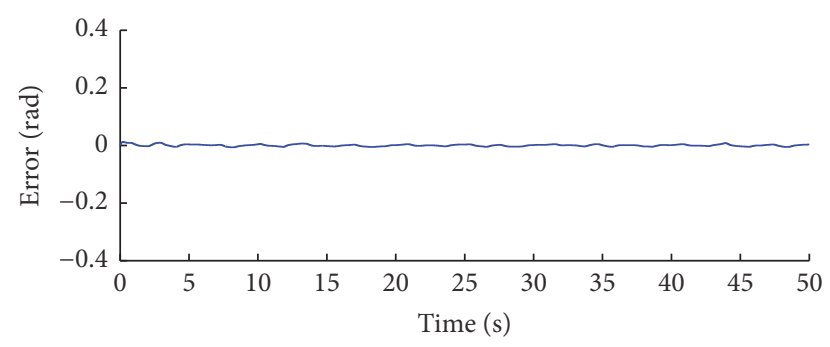

(b)

FIGURE 8: Simulation result of the designed adaptive laws for random trajectory case.

Figure 8 shows the result of the control law for a given random trajectory reference. Figure 8(a) illustrates the desired trajectory together with the simulated output for a random signal. The reference trajectory was generated using a uniform random generator filtered at $2 \mathrm{~Hz}$ cut-off frequency. Figure 8(a) shows the reference trajectory together with the controlled output, while Figure 8 (b) depicts the error of the trajectory. The proposed controller demonstrates a satisfactory performance as reflected by the error depicted in the lower panel of the same figure with $\mathrm{MSE}=9.8492 \times 10^{-5}$.

\section{Wind Tunnel Tests}

Three separate wind tunnel tests were performed. The FMW with a backstepping controller was tested in a gusty wind tunnel for robustness, and two different wind tunnel tests were performed to investigate the aerodynamic characteristics of the FMW mini-UAV. Steady uniform airflow was used to identify the aerodynamic properties of the FMW mini-UAV, while controlled gusty airflow was used to study the adaptive washout mechanism of the FMW and to validate the gust response dampening ability of the FMW mini-UAV.

6.1. Adaptive Controller Robustness Test. In order to validate the robustness of the backstepping controller and also to benchmark it with the feedforward/feedback controller in [10], an identical gusty wind tunnel test discussed in the same reference was performed on the FMW. Please refer to the reference for the description of the gust generator used in this experiment. Based on the dynamic analysis as shown in Figure 4, the modal parameters were identified, where the effective mass $m=0.17 \mathrm{~kg}, c=0.0026 \mathrm{Ns} / \mathrm{rad}$, and $k=$ $18.9 \mathrm{~N} / \mathrm{rad}$.

Figure 9 shows the results of the warping performance in two conditions, that is, no-gust and gusty environment. The left panels of the figure illustrate the performance of the feedforward/feedback controller (FF/PI), while the right ones show the performance of the system with the adaptive backstepping controller.

The backstepping controller is proven to be able to control the warping position when the FMW is subjected to disturbance. In case of minimum disturbance as simulated by the no-gust condition, the output of the system with FF/PI controller is able to track the reference input very well with RMS error of $0.285^{\circ}$. However, the performance slightly drops when the FMW is exposed under gusty environment (RMS $=0.523^{\circ}$ ). On the other hand, the adaptive backstepping controller performance seems to be more consistent for both cases, where the RMS errors are $0.447^{\circ}$ and $0.482^{\circ}$ for no-gust and gusty cases, respectively. Please note that, in this study, the parameters of the controllers are not fully optimized as the objective was more to validate the capability of using a controlled TSM in FMW applications rather than investigating the controller itself.

6.2. Aerodynamic Forces Characterization. The wind tunnel used for this experiment is $2.3 \mathrm{~m}$ wide, $1.5 \mathrm{~m}$ high, and $6 \mathrm{~m}$ long. A large test section was required in order to minimize the wall effects of the wind tunnel, which will affect the results of the wind tunnel tests. The FMW mini-UAV was tested at two different wind speeds, which were $12 \mathrm{~m} / \mathrm{s}$ and $15 \mathrm{~m} / \mathrm{s}$. Initially, the FMW mini-UAV was tested at a negative $\alpha$ of $-4^{\circ}$ and the whole wing fluttered aggressively. A similar incident occurred at $\alpha=0^{\circ}$, which results in the decision of testing the FMW at an angle of attack, $\alpha$, of $2^{\circ}$ onwards. The fluttering occurred because the FMW is rigid against forces from below the wing, but weak against the forces from above the wing. This property is expected, as it can allow the wing to provide lift while still being able to fold downwards around the fuselage. Even though the FMW was oscillating very aggressively, the TSM stayed intact and was able to still perform wing warping, proving its robustness in very harsh conditions.

The wind tunnel testing results are shown in Figures 10(a)-12(b). All the experimental results were taken three times at the same $\alpha$ and then averaged. The lift coefficient, $C_{L}$, drag coefficient, $C_{D}$, and pitching moment coefficient, $C_{M}$, were measured during the tests. The maximum $C_{L}$ of the FMW reached close to 1.5 when tested at $12 \mathrm{~m} / \mathrm{s}$ wind speed. From Figure 11, the highest $C_{L}$ over $C_{D}$ occurs when the angle of attack, $\alpha$, is around $8^{\circ}$ when the wind speed is $15 \mathrm{~m} / \mathrm{s}$. It can also be observed from the wind tunnel experiment that the rigid wing has a much lower stall angle, $\alpha$, when compared to the FMW. It is noted that, compared to the conventional rigid wing aircraft, the $C_{L} / C_{D}$ ratio of the FMW UAV is considerably small. This is due to the very small area of the wing, as well as the boxy frame of the fuselage structure, contributing to the high $C_{D}$ of the FMW UAV. Future versions of the FMW should have a more streamlined fuselage, with less wetted area in order to 

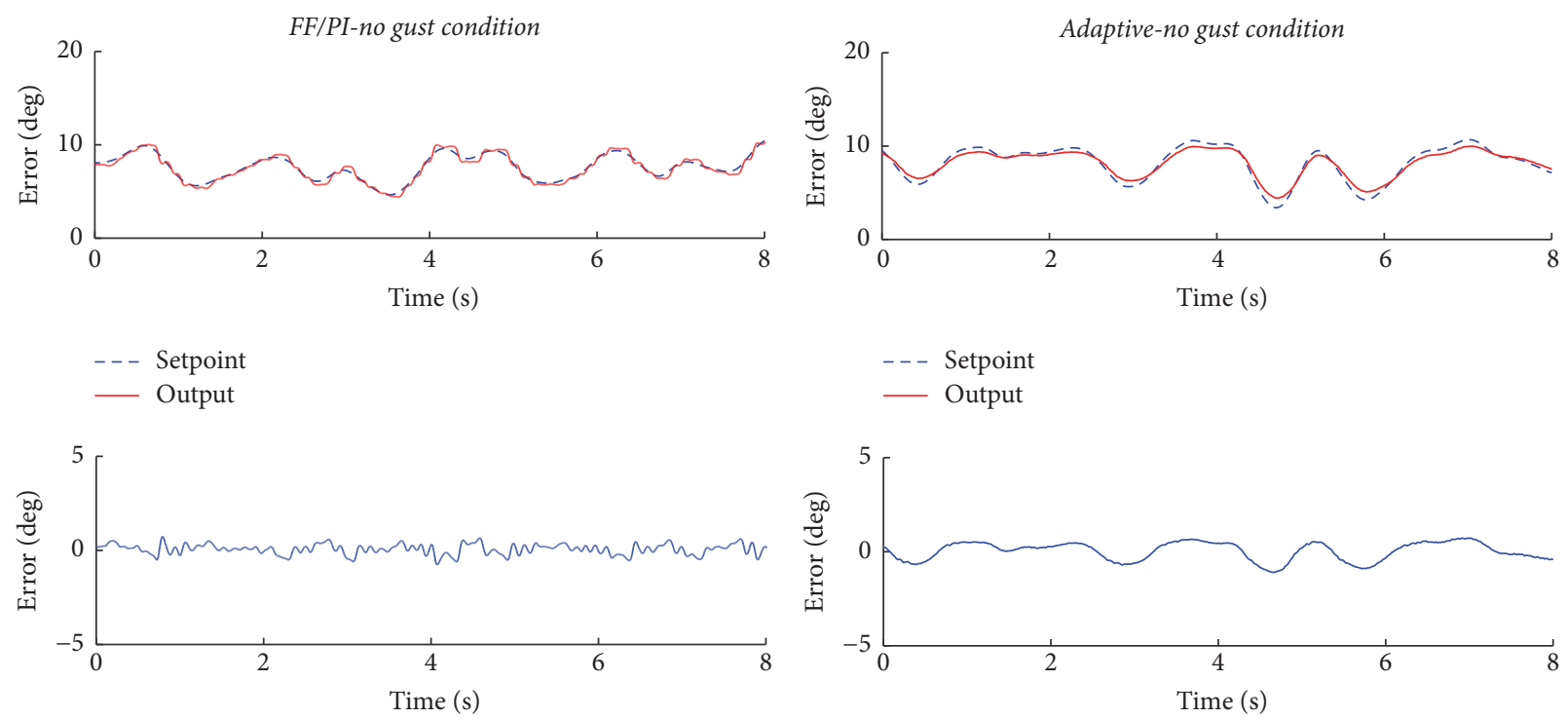

(a) Output and tracking error for no-gust condition
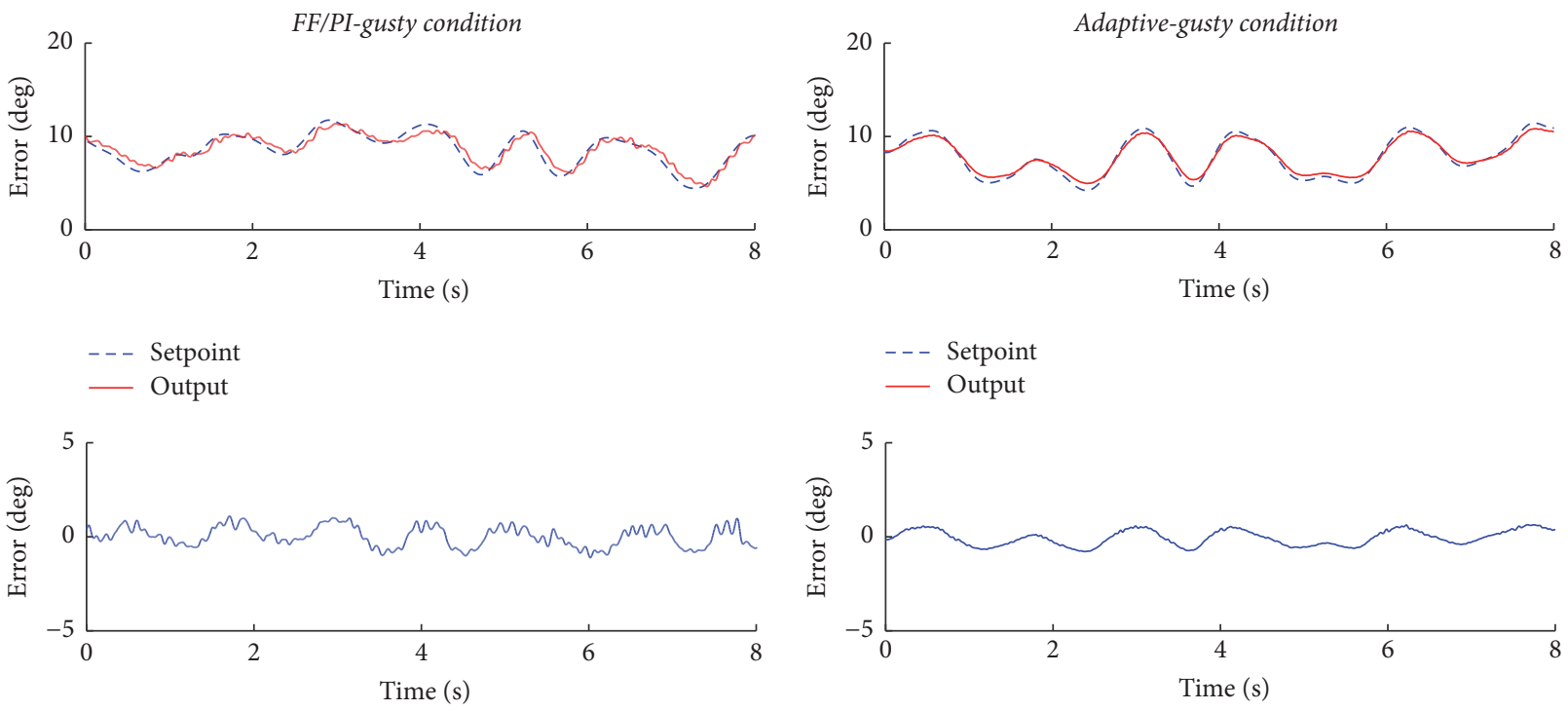

(b) Output and tracking error for gusty condition

FIGURE 9: Controller performances of the wing warping with tendon-sheath actuation under no-gust and gusty environments with wind speed of $7 \mathrm{~m} / \mathrm{s}$. The left panels show the case with feedforward/feedback controller; the right panels show the case of adaptive backstepping controller.

minimize the drag coefficient. The increase in wing area is desirable and will be more difficult to implement due to the material properties required of the FMW. Higher wing areas may lead to unwanted or unexpected folding. Further studies will be necessary to investigate the method of increasing the area as well as the aspect ratio of the FMW.

In Figure 12(a), the rigid wing stalls when $\alpha$ reaches $10^{\circ}$, while for the FMW the stall angle is at around $14^{\circ}$ when the wind speed is $15 \mathrm{~m} / \mathrm{s}$, and the FMW seems to not stall even at $18^{\circ}$ when the wind speed is $12 \mathrm{~m} / \mathrm{s}$. The rolling moment versus the warping angle is critical for the identification of the FMW roll rate and was obtained in this wind tunnel experiment. Holding the FMW at $\alpha=2^{\circ}$, Figure 12(b) shows that the rolling moment is increasing linearly with the warping angle, until warping angle reaches $14^{\circ}$, where the rolling moment starts to decrease. This phenomenon is quite well known as adverse rolling. For conventional wings, adverse rolling will happen when the ailerons are exerting too much force such that the wings itself are twisting into the opposite direction, creating the opposite rolling direction as intended from the pilot. In [24], the relationship between dynamic pressure and roll rate was investigated for rigid and flexible wings, and it was found that as the dynamic pressure increases, after a certain point, the flexible wings will lose the roll rate. For the FMW, the rolling moment starts to decrease when the warping angle is beyond $14^{\circ}$ at $15 \mathrm{~m} / \mathrm{s}$. Therefore, for wind 


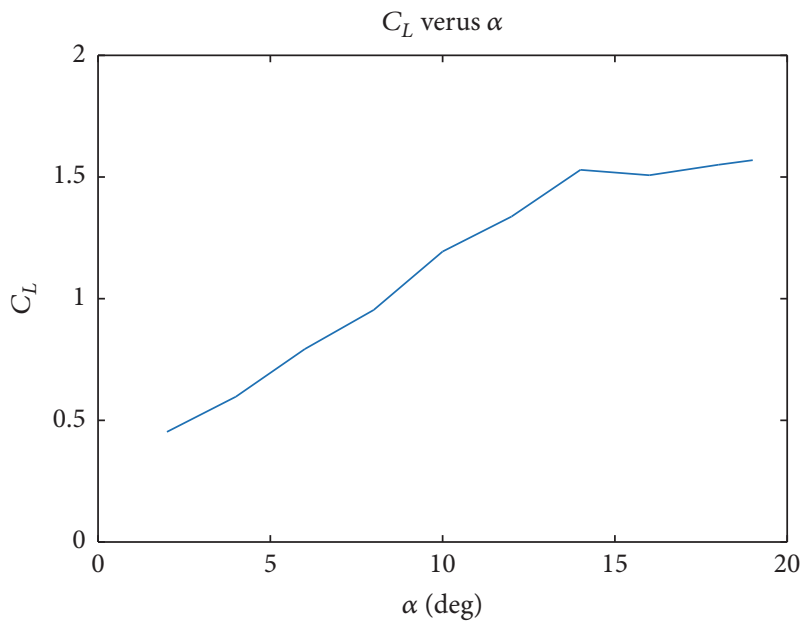

(a) $C_{L}$ versus $\alpha(\operatorname{deg})$ at $12 \mathrm{~m} / \mathrm{s}$

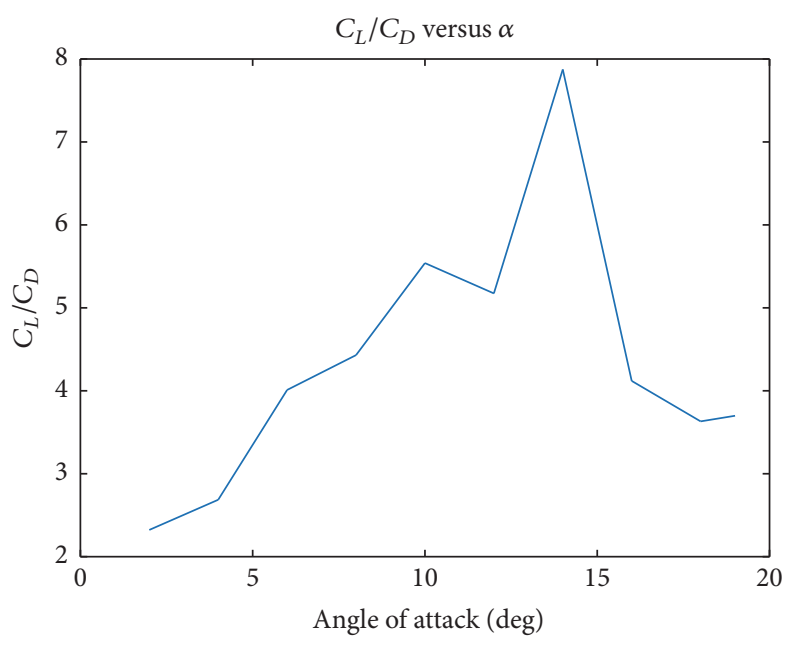

(c) $C_{L}$ over $C_{D}$ versus $\alpha(\mathrm{deg})$ at $12 \mathrm{~m} / \mathrm{s}$

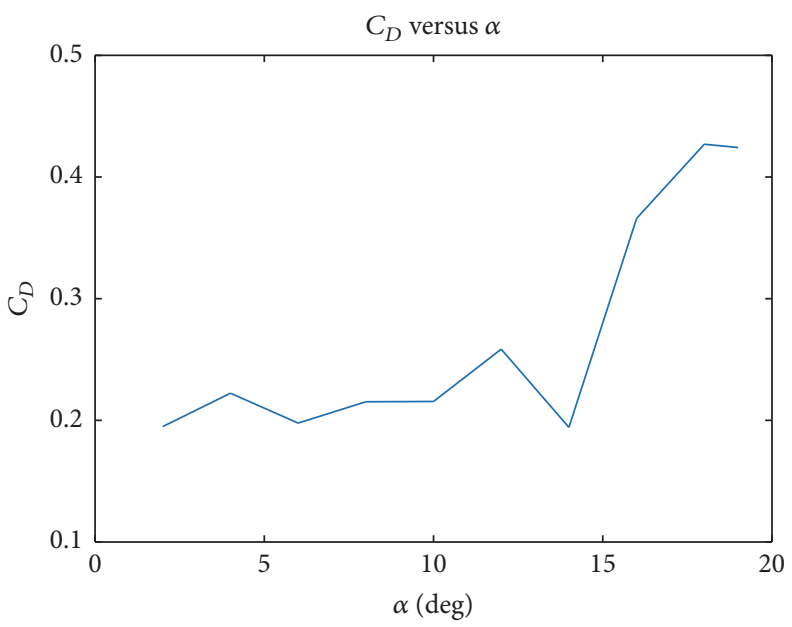

(b) $C_{D}$ versus $\alpha(\mathrm{deg})$ at $12 \mathrm{~m} / \mathrm{s}$

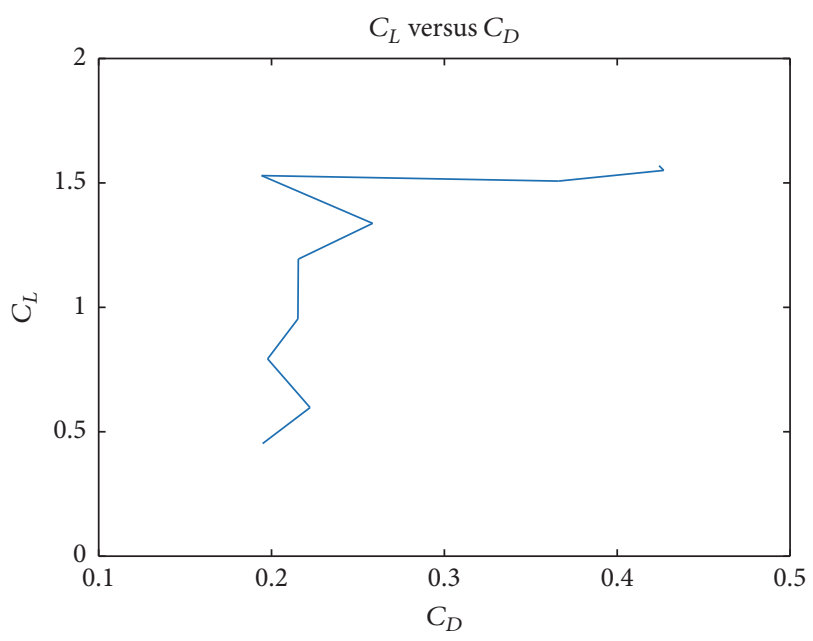

(d) $C_{L}$ versus $C_{D}$ at $12 \mathrm{~m} / \mathrm{s}$

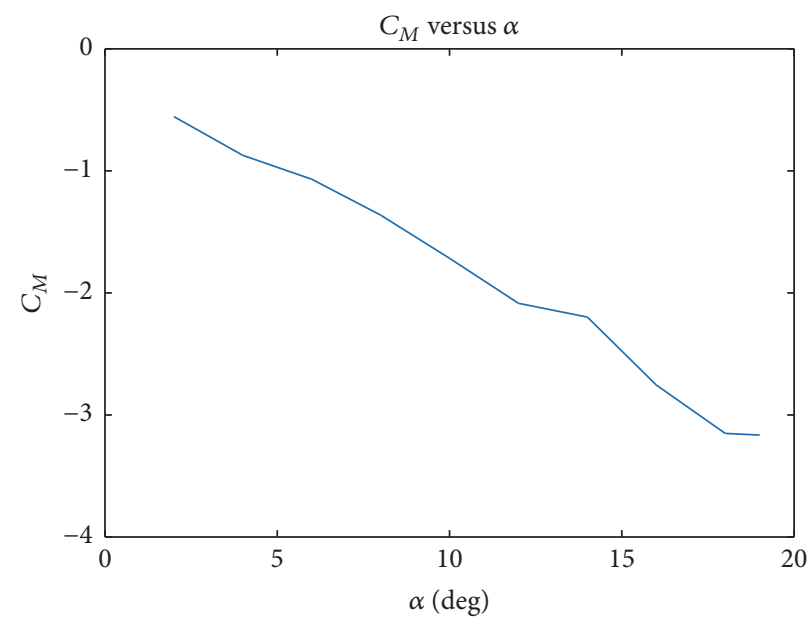

(e) $C_{M}$ versus $\alpha(\mathrm{deg})$ at $12 \mathrm{~m} / \mathrm{s}$

Figure 10: Aerodynamic properties of the FMW mini-UAV at $12 \mathrm{~m} / \mathrm{s}$ wind speed. 


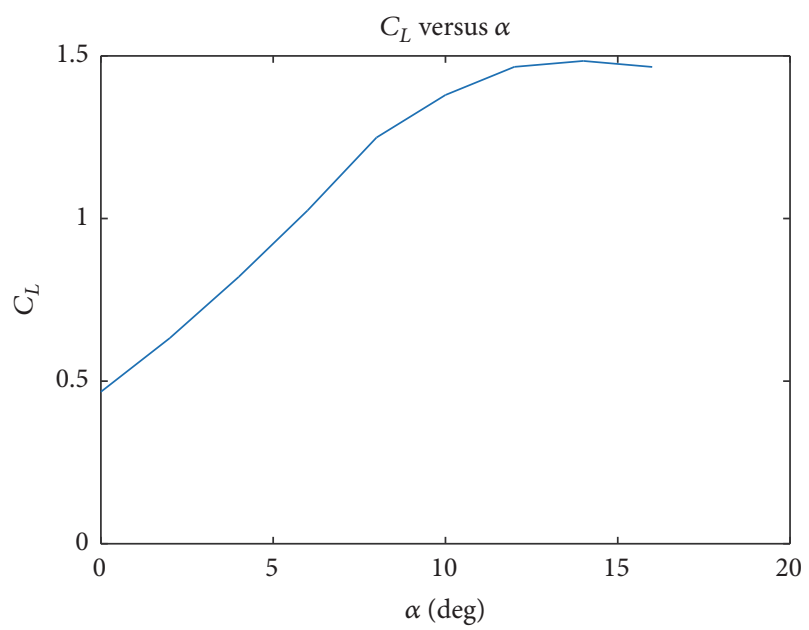

(a) $C_{L}$ versus $\alpha(\mathrm{deg})$ at $15 \mathrm{~m} / \mathrm{s}$

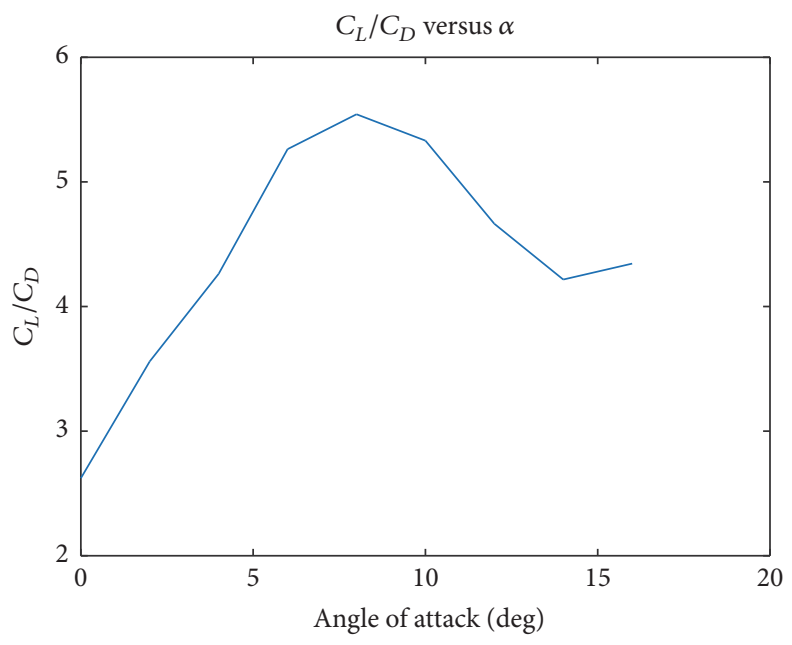

(c) $C_{L}$ over $C_{D}$ versus $\alpha(\mathrm{deg})$ at $15 \mathrm{~m} / \mathrm{s}$

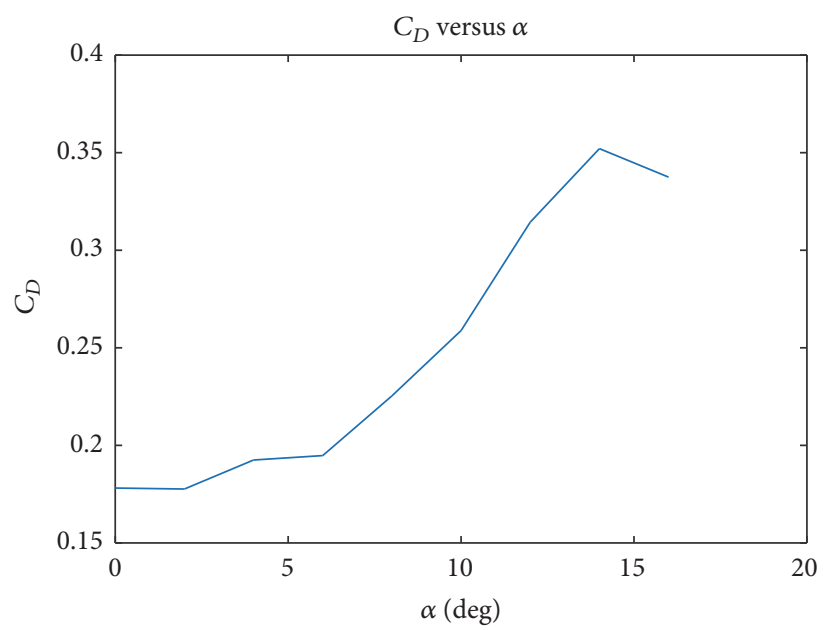

(b) $C_{D}$ versus $\alpha(\mathrm{deg})$ at $15 \mathrm{~m} / \mathrm{s}$

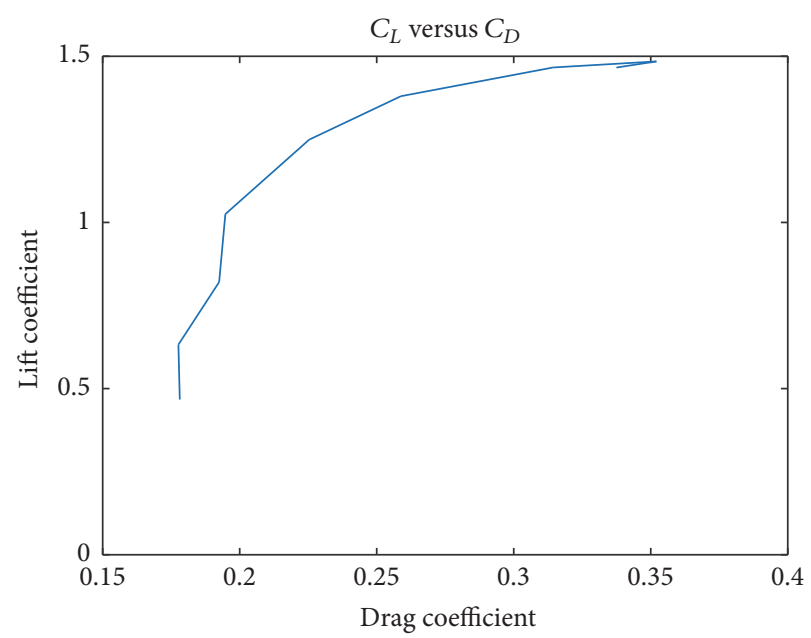

(d) $C_{L}$ versus $C_{D}$ at $15 \mathrm{~m} / \mathrm{s}$

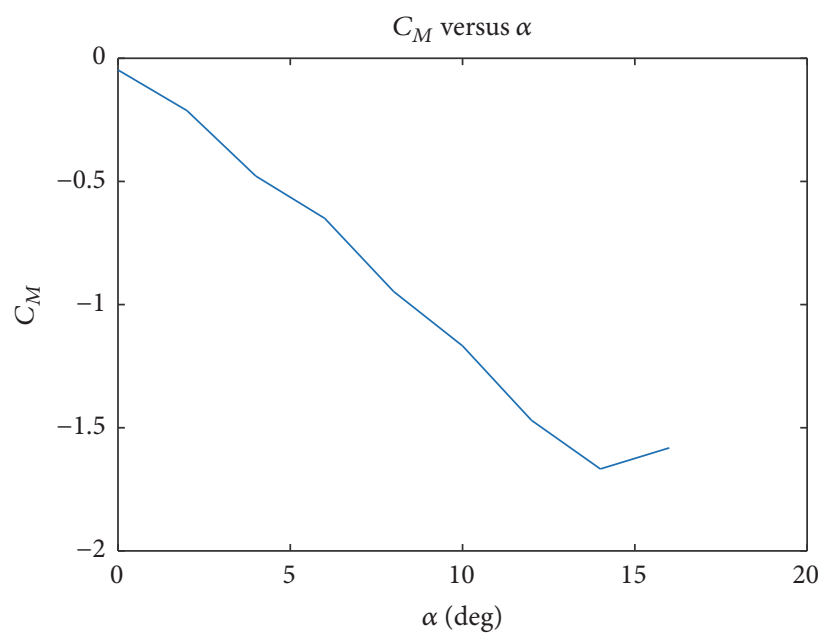

(e) $C_{M}$ versus $\alpha(\operatorname{deg})$ at $15 \mathrm{~m} / \mathrm{s}$

FIGURE 11: Aerodynamic properties of the FMW mini-UAV in $15 \mathrm{~m} / \mathrm{s}$ wind speed. 


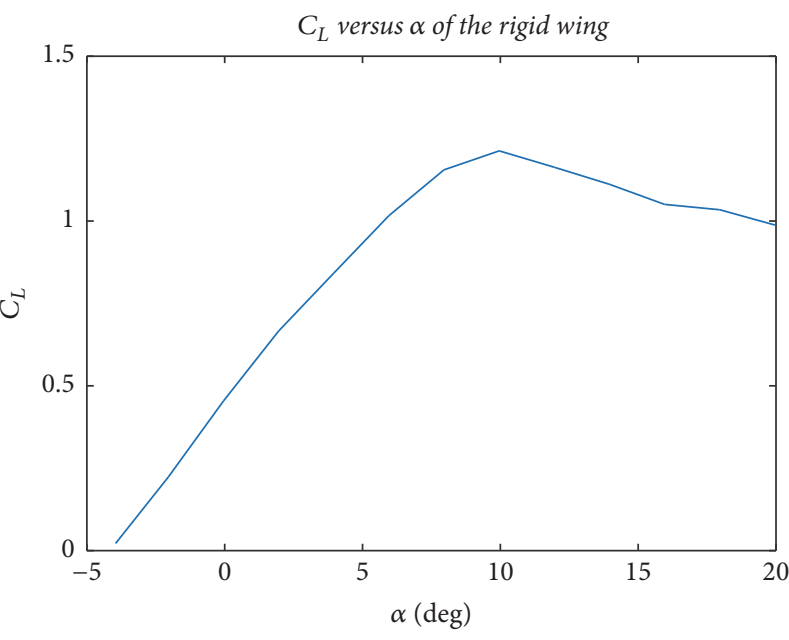

(a)

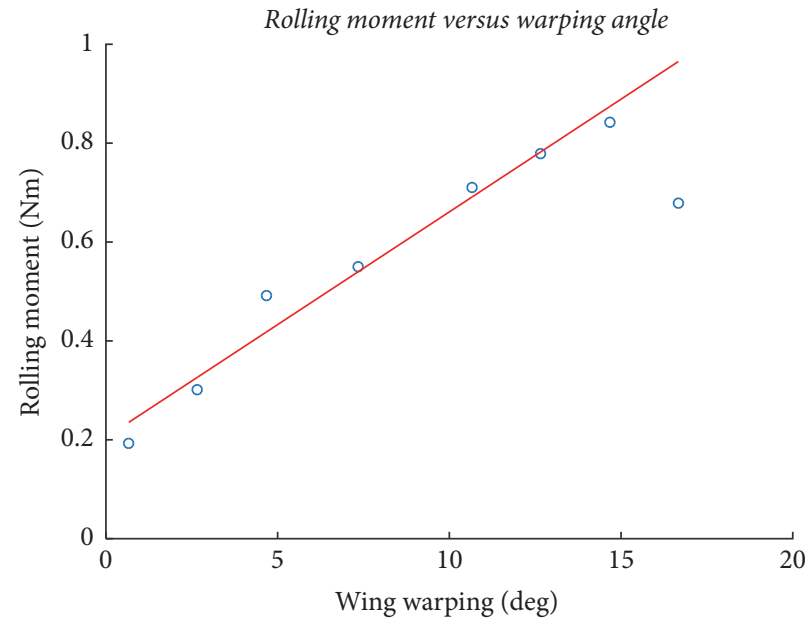

(b)

FIGURE 12: Wind tunnel test results: (a) aerodynamic property of rigid wing; (b) the rolling moment of FMW.

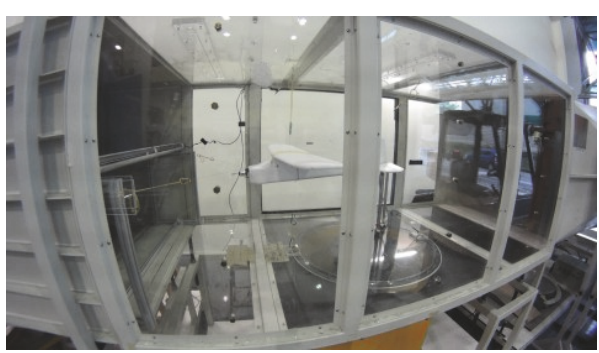

(a)

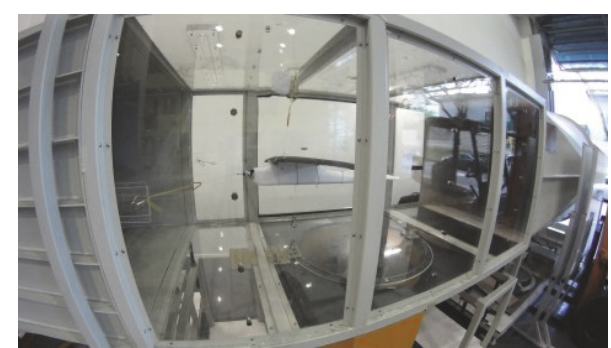

(b)

FIGURE 13: Test rigs: (a) the rigid wing and (b) the FMW model, suspended in the middle of the test section of the wind tunnel.

speeds larger than $15 \mathrm{~m} / \mathrm{s}$, it is undesirable to warp the FMW more than $14^{\circ}$. Further investigations are required to map the relationship between the roll rate, warping angle, and also the dynamic pressure for the FMW.

6.3. Adaptive Washout Validation. One of the capabilities of the FMW is to dampen the longitudinal gust response via the adaptive washout mechanism. When a longitudinal direction gust hits the FMW from below, it will create an extra lift force, but the lift force will be dampened as the FMW will flex chordwise passively, changing the airfoil shape and also the effective $\alpha$ [3]. Thus, the gust response of the FMW is much lower compared to a conventional rigid wing. When the TSM is installed on the FMW, a validation of the adaptive washout is required. A gusty flow wind tunnel test is performed to obtain data about the gust response of both the FMW miniUAV and the conventional rigid wing mini-UAV to validate the adaptive washout mechanism of the FMW when installed with the tendon-sheath.

The mini-UAV test model was suspended inside the wind tunnel, with unconstrained pitch and roll degrees-offreedom, as well as freedom of translational movement along the three axes. Nylon strings were used to suspend the model, and rubber bands were tied to the end of the nylon strings to help increase the deflection and rotational angles of the model. The nylon strings were attached to the nose and the tail of the model, as well as at the aerodynamic center of the model to support the weight of the model. The static margin of the model was set to $5 \%$ for a more sensitive response to gusts. The model was fitted with all the payload, with a simulated weight to mimic all electronics and battery suspended in the wind tunnel to obtain a close approximate of the moment of inertias and also to better simulate a real flight situation. Figure 13 illustrates two models, with rigid wings-as a comparison-and FMW, suspended in the test section of the wind tunnel.

To generate longitudinal gusts, a rotating slotted cylinder (RSC) device was used. The RSC is a simple mechanism, which utilizes only one rotating cylinder to generate the simulated gusts. The RSC was designed following [25, 26], where the fundamental frequency of the generated gust is equal to two times the rotational frequency of the cylinder. The chord length of the airfoil, $c$, in the gust generator is $0.2 \mathrm{~m}$, and the diameter of the RSC is $d=0.25 c$. The thickness of the RSC is $t=0.0313 c$ and the gap between the airfoil trailing edge and the RSC is $e=0.08 c$. NACA 0012 was used as the airfoil for the gust generator. The RSC gust generator is shown in Figure 14. The frequency of the gust is set at $7 \mathrm{~Hz}$ as this is 
TABLE 2: Specifications of the FMW mini-UAV.

\begin{tabular}{lc}
\hline Wingspan & $1 \mathrm{~m}$ \\
CG location & $0.25 \mathrm{~m}$ behind leading edge \\
Weight & $1060 \mathrm{~g}$ \\
Wing loading & $53 \mathrm{~g} / \mathrm{dm}^{3}$ \\
Power & Electric motor $+9^{\prime \prime} \times 6^{\prime \prime}$ propellers \\
Estimated flight time & 8 minutes \\
\hline
\end{tabular}

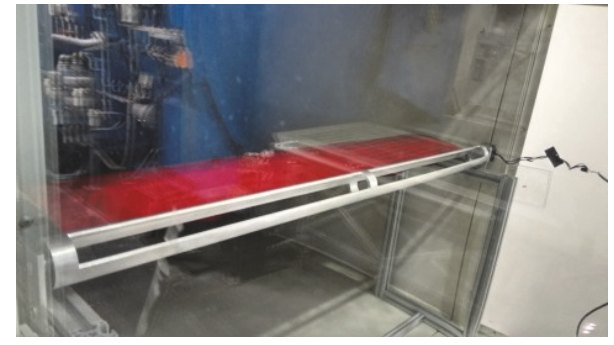

FIGURE 14: Gust generator with a rotating slotted cylinder.

the fundamental frequency of the FMW. Therefore, the RSC rotates at 3.5 revolutions per second.

The model was fitted with accelerometers and gyroscopes at the center of gravity to detect the movements of the model, and the oscillation data is then streamed and recorded to a data acquisition computer. Figure 15 shows the gust response of the rigid wing and FMW model. From the plots it can be observed that the adaptive washout mechanism of the FMW helped to alleviate the gust response, especially in the $z$-axis (see Figures 15(e) and 15(f)), as the FMW model has less movements in the gusty airflow compared to the rigid conventional wing model. The standard deviation of the rigid wing movements is generally larger compared to the FMW, with the most significant difference in the $z$-axis acceleration at 214.82 for the rigid wing and 94.17 for the FMW.

\section{Flight Test}

To provide autopilot control to the aircraft and to track the flight data, the ArduPilot (APM) 2.5 flight controller is fitted at the CG of the FMW mini-UAV. The main control strategy used for the autopilot is PID feedback for all the flight dynamics. The STM32F4 microcontroller preprogrammed with the control strategy presented in Section 5 is also used to regulate the wing warping and all the sensors and actuators related to the wing warping are connected to this microcontroller. The specifications of the UAV are listed in Table 2.

As the relationship between the rolling moment and the warping position can be modeled as a linear curve from wind tunnel tests, a PID feedback control will be sufficient. From the wind tunnel tests, roll reversal of the FMW has been found at warping angle $14^{\circ}$ at $15 \mathrm{~m} / \mathrm{s}$ wind speed. In this flight test, the wing warping has been saturated at $14^{\circ}$ in order to avoid adverse rolling of the mini-UAV. In the future, further investigation will be needed to examine the relationship between rolling moment and dynamic pressure of the FMW.

Figure 16 shows the mini-UAV cruising in the sky. Flying the FMW mini-UAV requires a good piloting skill as the aircraft is very fast and responsive. Therefore, in this following discussion, the flight controllability and maneuverability are assessed qualitatively based on the input from the pilot. According to the pilot, the mini-UAV is very difficult to control without wing morphing strategy (when maneuvering relies only to the rudders), as it is a fast aircraft. There is more control on the rolling movement in a case with the controlled wing warping mechanism, compared to that with only using the rudder input. Rudders are able to provide yaw and banking control only at low speeds, that is, during gliding. During all the flight tests the weather was gusty. Maneuvering the aircraft with an open-loop wing morphing mechanism does not give acceptable performance, either. The aircraft was barely under control. This result is believed to be attributed to the incapability of an open-loop system to manage disturbances. However, the mini-UAV was able to fly steadily under control with the controlled wing warping mechanism.

Figures 17, 18, and 19 illustrate the flight test results to give qualitative measures of the mini-UAV responsiveness under three different control methods, that is, (i) relying only on the rudder input (with no wing warping), (ii) open-loop wing warping, and (iii) backstepping controlled wing warping, to achieve rolling motion for the aircraft. Even though the tests were not conducted exactly at the same instance, those were conducted successively within short duration to minimize the variation of environmental conditions (e.g., gust).

Figures 17(a), 18(a), and 19(a) show the reference input given through the UAV remote controller, while Figures 17(b), 18(b), and 19(b) show the roll rates results. It can be seen easily from the three figures that the wing warping mechanism with the proposed controller demonstrates the best qualitative performance; that is, the output tends to follow the reference input. Rudder input can also induce rolling; however, it is not as effective as the controlled wing warping.

Figure 20 shows the cross correlation between the wing warping input and the roll rate output for the third case (FMW with the controller). It can be seen that the output has a strong correlation to the reference input with an acceptable lag of 0.3 seconds. In gusty condition during the flight test, the mini-UAV was able to achieve a 0.7625 correlation coefficient between the wing warping input and the roll rate. The openloop wing warping control did not provide a sufficient control of the rolling moment of the mini-UAV, as experienced during flights and also shown in the flight data, with a correlation coefficient of only 0.0945 . The rudder inputs did not seem to affect the roll rates of the aircraft either, with the correlation coefficient at 0.1347 .

\section{Discussion and Conclusion}

An FMW was designed to dampen gusts while in flight and also to fold around the fuselage for storage. However, the FMW lacks a suitable actuation method to be able to provide ample rolling moment. A TSM is proven to be a suitable 


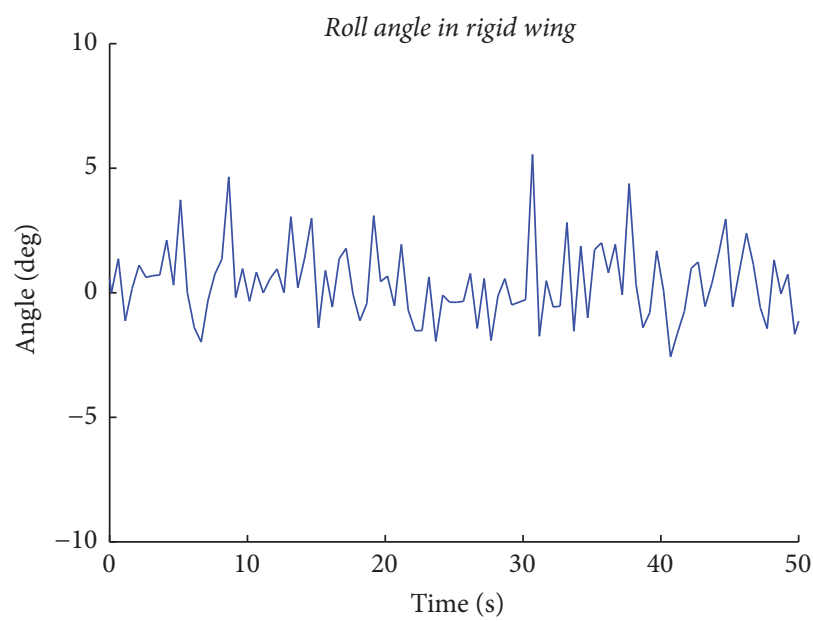

(a)

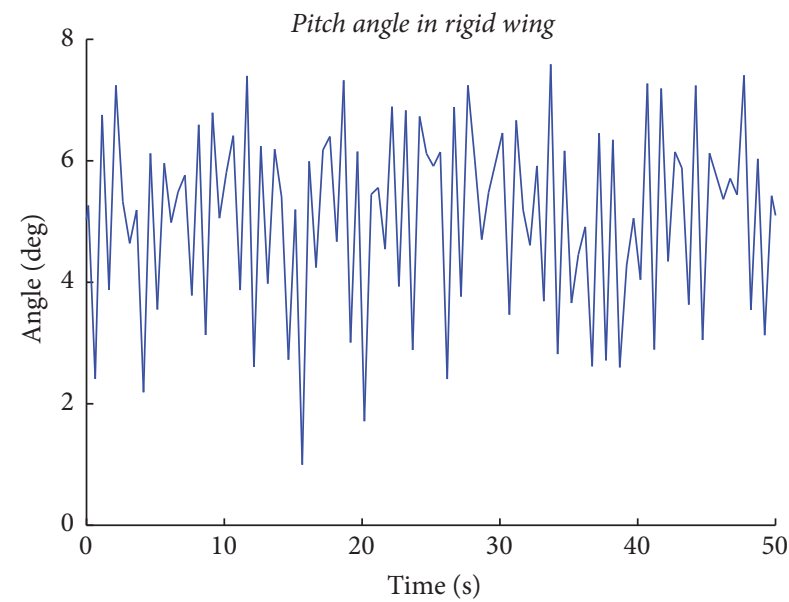

(c)

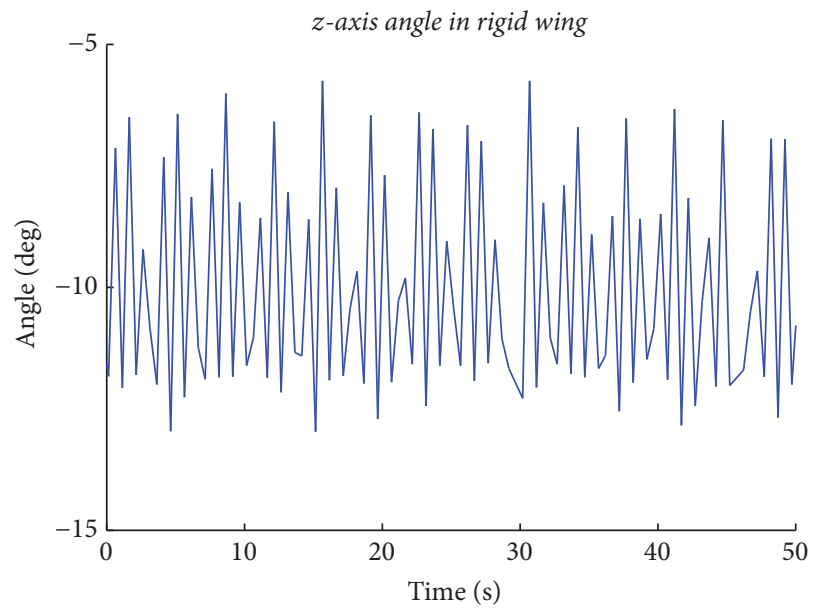

(e)

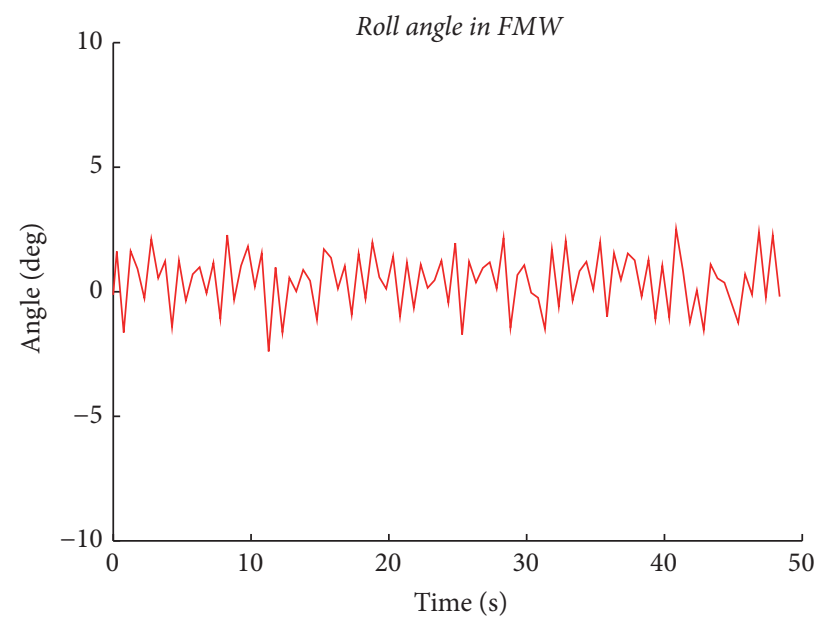

(b)

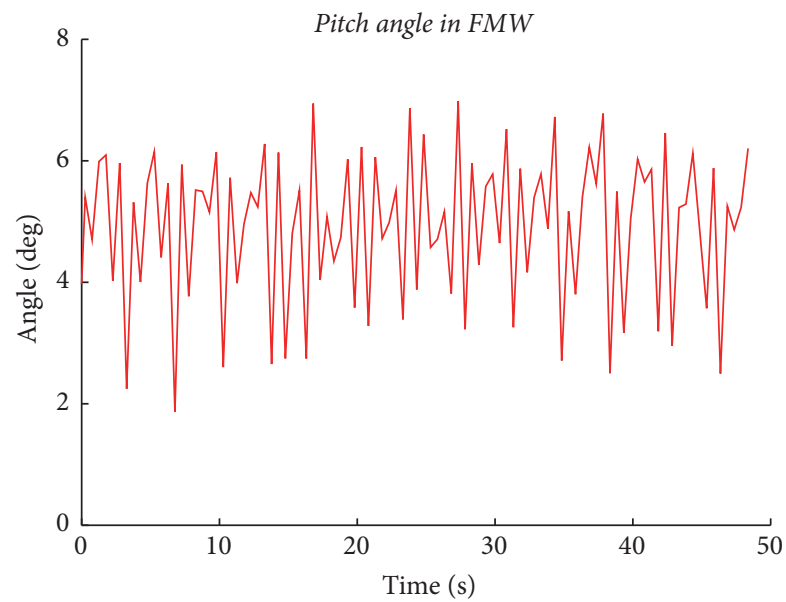

(d)

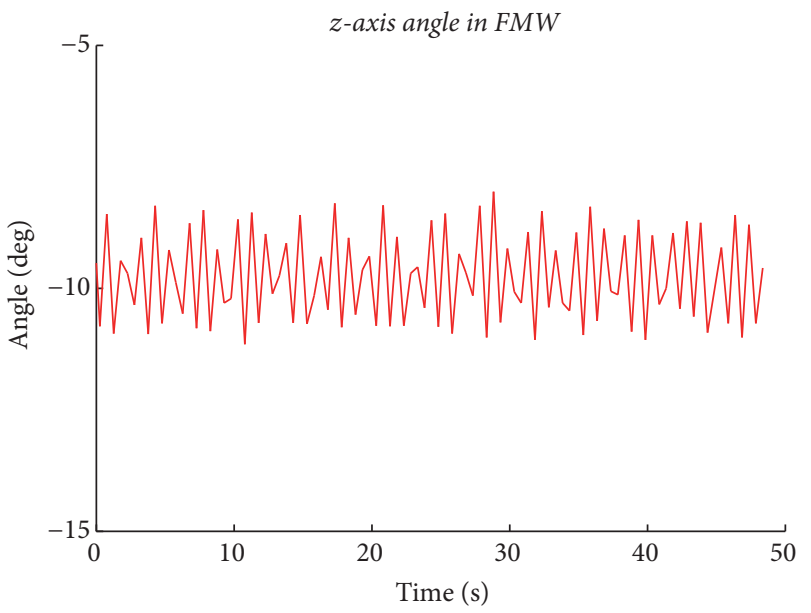

(f)

FIGURE 15: The roll and pitch angles and $z$-axis acceleration of both the rigid wing and FMW model at $7 \mathrm{~Hz}$ longitudinal gusts. Rigid wing behavior is shown in (a), (c), and (e), while those from FMW are shown in (b), (d), and (f). 
candidate to provide the needed actuation without sacrificing the desired attributes of the FMW.

In this paper, an adaptive backstepping controller was proposed, and it was concluded that the controller is more robust to the disturbance compared to a model-based feedforward controller. Furthermore, a mini-UAV was designed and various tests were performed to identify its characteristics. From the wind tunnel tests, the FMW installed with TSM demonstrated the ability of the adaptive washout, being able to dampen the longitudinal gusts quite well when compared to a rigid, conventional wing. It was also shown that the rudder only input does not induce as much rolling moment as the controlled wing warping. Flight tests were conducted, and the wing warping was proven to be a better method to induce rolling moment when compared to rudder input only. The TSM actuated FMW with the proposed control strategy was proven to work well in a real flight.

One of the findings from the wind tunnel experiments was the roll reversal of the FMW. Due to the flexibility of the wing in the chordwise direction, roll reversal will exist when the dynamic pressure is very high. Careful saturation of the FMW warping is necessary for the safety of the aircraft. Future work could characterize the occurrence of roll reversal in different situations and develop a better control strategy to prevent it.

\section{Appendix}

\section{Proof of Theorem 2}

Define a Lyapunov function candidate, $V$, as follows:

$$
V=\frac{\xi_{1}^{2}}{2}+\frac{\xi_{2}^{2}}{2}+\frac{\widetilde{\Theta}^{T} \widetilde{\Theta}}{2 k_{\Theta}}+\frac{\widetilde{m}^{2}}{2 m k_{m}}+\frac{\left(\widetilde{D}^{*}\right)^{2}}{2 m k_{D}} .
$$

Taking the derivative of $V$ together with (14) and (21), we can obtain

$$
\begin{aligned}
\dot{V}= & \xi_{1} \dot{\xi}_{1}+\xi_{2} \dot{\xi}_{2}-\frac{\widetilde{\Theta}^{T} \dot{\widehat{\Theta}}}{k_{\Theta}}-\frac{\widetilde{m} \dot{\bar{m}}}{m k_{m}}-\frac{\widetilde{D}^{*} \dot{\widehat{D}}^{*}}{m k_{D}} \\
= & \xi_{1}\left(\xi_{2}-\alpha_{v 1} \xi_{1}\right) \\
& +\xi_{2}\left(\frac{1}{m}(u+D)+\Theta^{T} \varphi-\ddot{y}_{r}+\alpha_{v 1} \dot{\xi}_{1}\right)-\frac{\widetilde{m} \dot{\widehat{m}}}{m k_{m}} \\
& -\frac{\widetilde{\Theta}^{T} \dot{\Theta}}{k_{\Theta}}-\frac{\widetilde{D}^{*} \dot{\vec{D}}^{*}}{m k_{D}} .
\end{aligned}
$$

Substituting (17) and (18), we can rewrite (A.2) as

$$
\begin{aligned}
\dot{V}= & -\alpha_{v 1} \xi_{1}^{2}-\alpha_{v 2} \xi_{2}^{2}+\left(\widetilde{\Theta}^{T} \varphi \xi_{2}-\frac{\widetilde{\Theta}^{T} \dot{\Theta}}{k_{\Theta}}\right) \\
& -\left(\frac{\widetilde{m} \bar{u} \xi_{2}}{m}+\frac{\widetilde{m} \dot{\bar{m}}}{m k_{m}}\right) \\
& +\left(\frac{D \xi_{2}}{m}-\frac{\widetilde{D}^{*} \dot{\widehat{D}}^{*}}{m k_{D}}-\frac{\widehat{D}^{*} \xi_{2} \tanh \left(\xi_{2} / \varepsilon\right)}{m}\right) .
\end{aligned}
$$

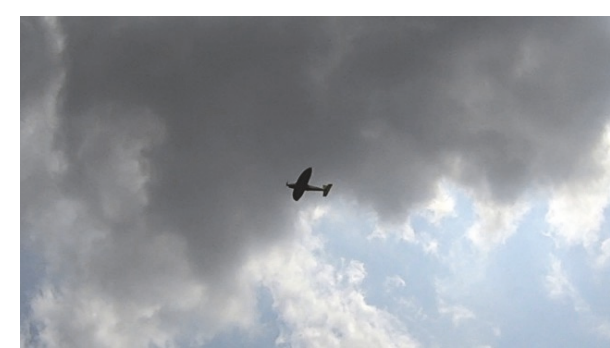

FIGURE 16: Flight test of the FMW mini-UAV.

From (19) to (21), the following expression is observed:

$$
\begin{aligned}
& \widetilde{\Theta}^{T} \varphi \xi_{2}-\frac{\widetilde{\Theta}^{T} \dot{\widehat{\Theta}}}{k_{\Theta}}=\widetilde{\Theta}^{T} \varphi \xi_{2}-\frac{\widetilde{\Theta}^{T}\left(k_{\Theta} \xi_{2} \varphi-\sigma_{1} \widehat{\Theta}\right)}{k_{\Theta}} \\
& =\frac{\sigma_{1} \widetilde{\Theta}^{T} \widehat{\Theta}}{k_{\Theta}}, \\
& \frac{\widetilde{m} \bar{u} \xi_{2}}{m}+\frac{\widetilde{m} \dot{\bar{m}}}{m k_{m}}=\frac{\widetilde{m} \bar{u} \xi_{2}}{m}-\frac{\widetilde{m}\left(k_{m} \xi_{2} \bar{u}+\sigma_{2} \widehat{m}\right)}{m k_{m}} \\
& =-\frac{\sigma_{2} \widetilde{m} \widehat{m}}{m k_{m}}, \\
& \frac{\widetilde{D}^{*} \dot{\widehat{D}}^{*}}{m k_{D}}=\frac{\left(D^{*}-\widehat{D}^{*}\right) k_{D} \xi_{2} \tanh \left(\xi_{2} / \varepsilon\right)}{m k_{D}}-\frac{\sigma_{3} \widetilde{D}^{*} \widehat{D}^{*}}{m k_{D}} .
\end{aligned}
$$

Subsequently, (A.3) can be rewritten as

$$
\begin{aligned}
\dot{V}= & -\alpha_{v 1} \xi_{1}^{2}-\alpha_{v 2} \xi_{2}^{2}+\frac{\sigma_{1} \widetilde{\Theta}^{T} \widehat{\Theta}}{k_{\Theta}}+\frac{\sigma_{2} \widetilde{m} \widehat{m}}{m k_{m}}+\frac{\sigma_{3} \widetilde{D}^{*} \widehat{D}^{*}}{m k_{D}} \\
& +\frac{D \xi_{2}}{m}-\frac{\widehat{D}^{*} \xi_{2} \tanh \left(\xi_{2} / \varepsilon\right)}{m} .
\end{aligned}
$$

Applying Young's inequality for two numbers $a$ and $b$, that is, $a b \leq 0.5\left(a^{2}+b^{2}\right)$, the third, fourth, and fifth terms in right hand side of (18) can be reformulated as

$$
\begin{aligned}
\frac{\sigma_{1} \widetilde{\Theta}^{T} \widehat{\Theta}}{k_{\Theta}} & =\frac{\sigma_{1} \widetilde{\Theta}^{T}(\Theta-\widetilde{\Theta})}{k_{\Theta}} \leq \frac{\sigma_{1} \Theta^{T} \Theta}{2 k_{\Theta}}-\frac{\sigma_{1} \widetilde{\Theta}^{T} \widetilde{\Theta}}{2 k_{\Theta}}, \\
\frac{\sigma_{2} \widetilde{m} \widehat{m}}{m k_{m}} & =\frac{\sigma_{2} \widetilde{m}(m-\widetilde{m})}{m k_{m}} \leq \frac{\sigma_{2} m^{2}}{2 m k_{m}}-\frac{\sigma_{2} \widetilde{m}^{2}}{2 m k_{m}}, \\
\frac{\sigma_{3} \widetilde{D}^{*} \widehat{D}^{*}}{m k_{D}} & =\frac{\sigma_{3} \widetilde{D}^{*}\left(D^{*}-\widetilde{D}^{*}\right)}{m k_{D}} \\
& \leq \frac{\sigma_{3} D^{* 2}}{2 m k_{D}}-\frac{\sigma_{3}\left(\widetilde{D}^{*}\right)^{2}}{2 m k_{D}} .
\end{aligned}
$$

From (A.6) and using a proven property in [27], $\tanh \left(\xi_{2} / \varepsilon\right)$ obeys $\left|\xi_{2}\right|-\xi_{2} \tanh \left(\xi_{2} / \varepsilon\right) \leq 0.2785 \varepsilon$. 


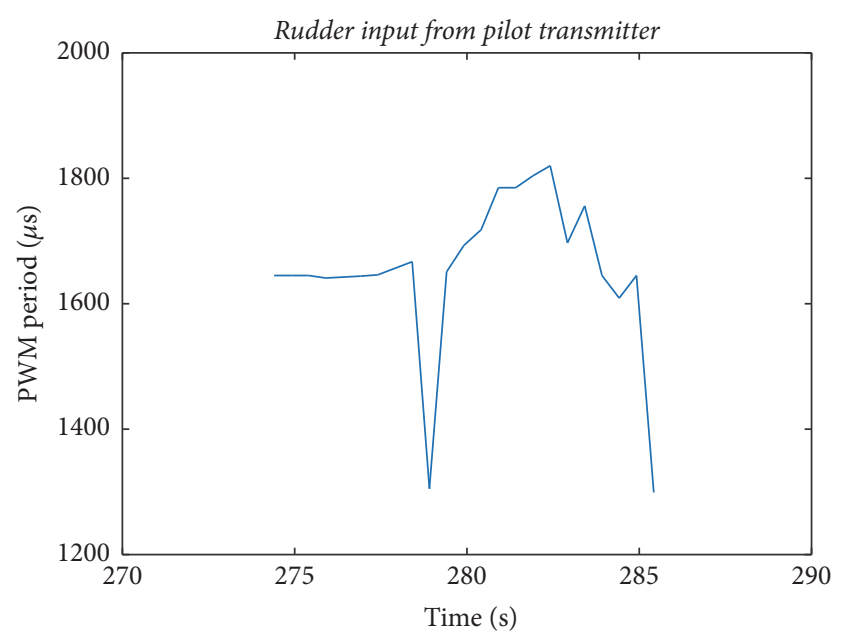

(a)

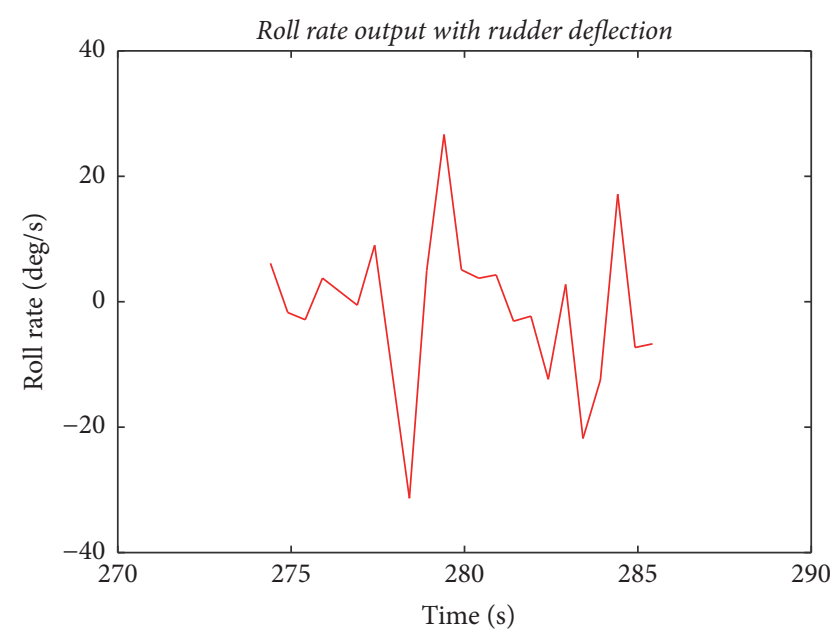

(b)

FIGURE 17: Roll rate of the FMW when given rudder doublets: (a) rudder inputs from pilot; (b) roll rates of the FMW mini-UAV.

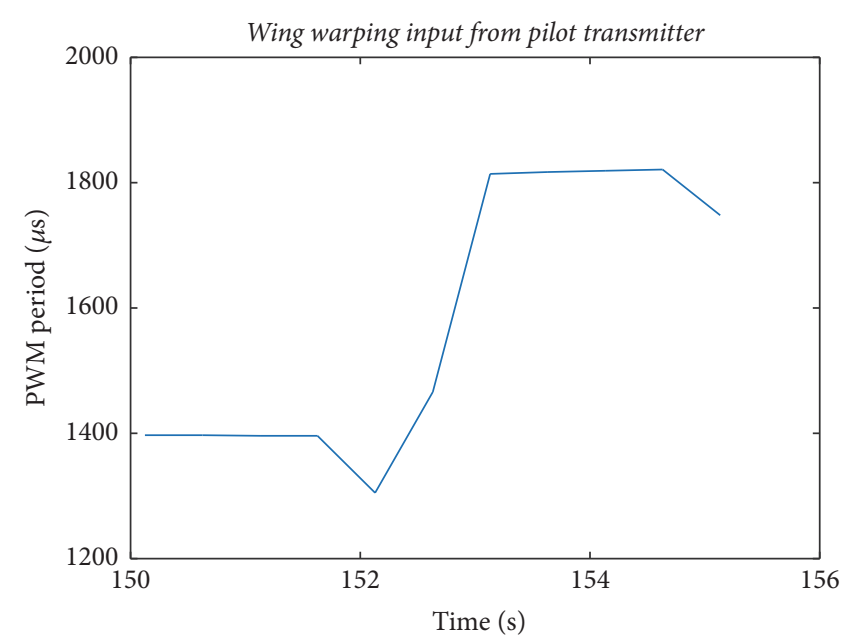

(a)

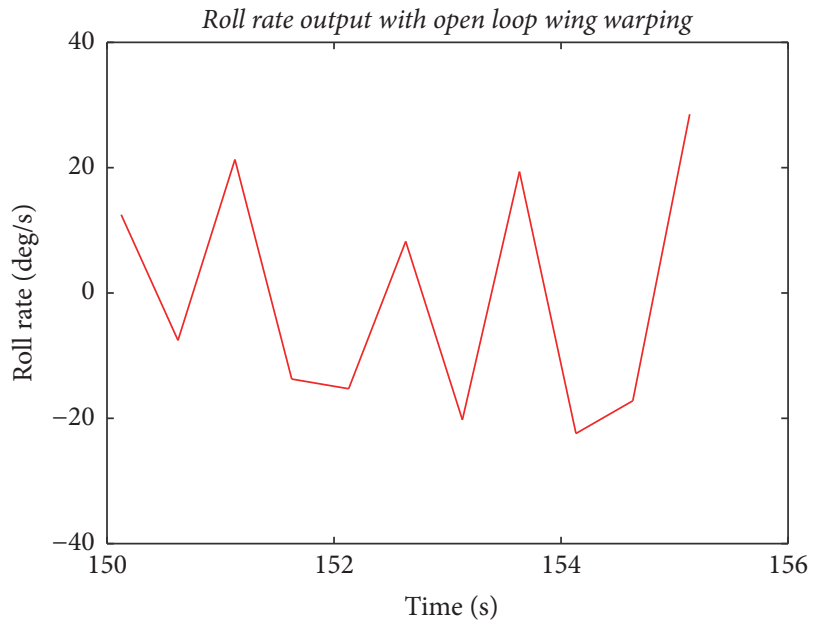

(b)

FIGURE 18: Roll rate of the FMW with open-loop wing warping double: (a) rudder inputs from pilot; (b) roll rates of the FMW mini-UAV.

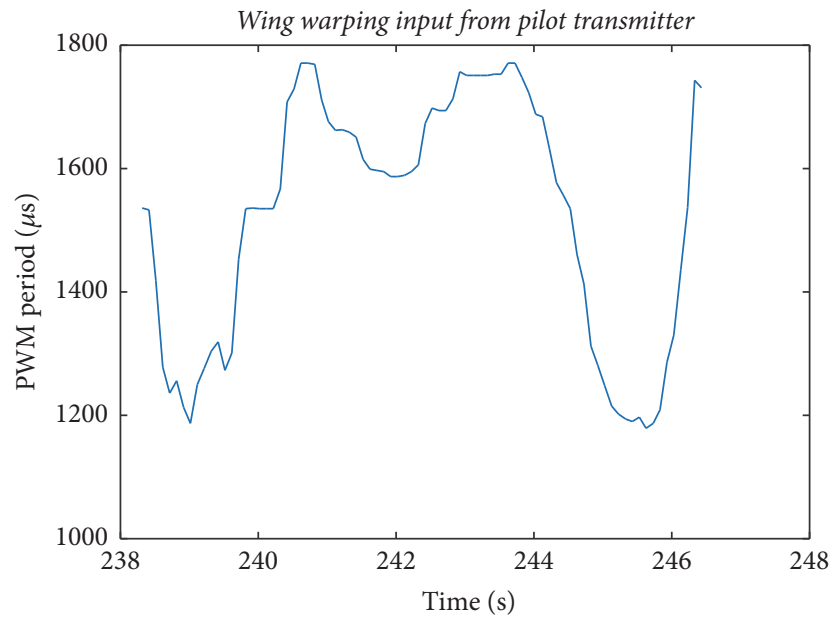

(a)

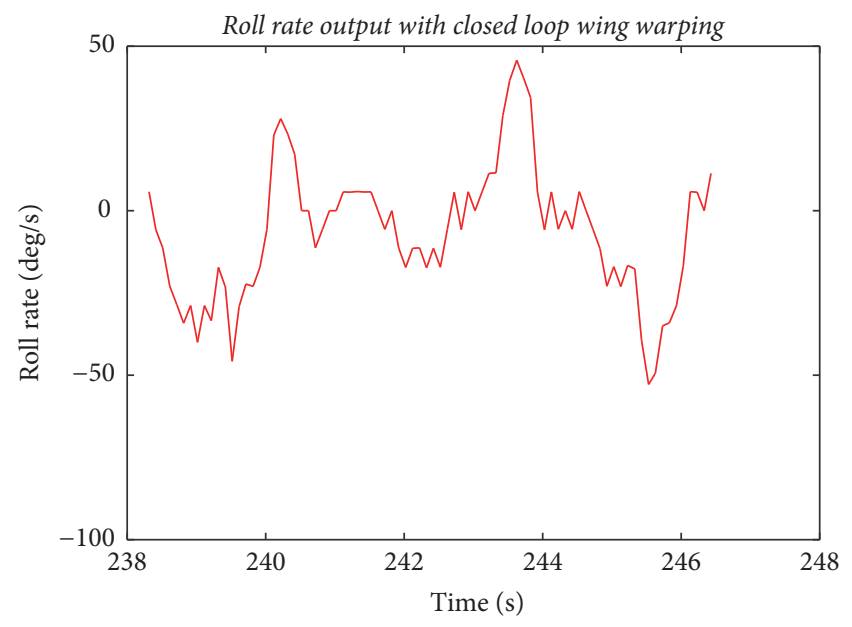

(b)

FIGURE 19: Roll rate of the FMW with closed-loop wing warping double: (a) rudder inputs from pilot; (b) roll rates of the FMW mini-UAV. 


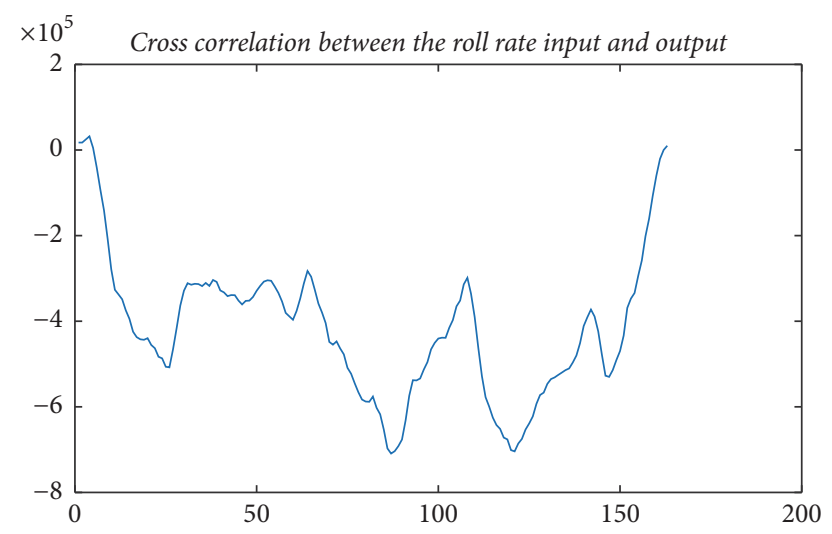

FIGURE 20: Cross correlation between the wing warping input and roll rate output of the FMW mini-UAV with controller.

$\dot{V}$ in (A.5) can be rewritten as

$$
\begin{aligned}
\dot{V} \leq & -\alpha_{v 1} \xi_{1}^{2}-\alpha_{v 2} \xi_{2}^{2}-\frac{\sigma_{1} \widetilde{\Theta}^{T} \widetilde{\Theta}}{2 k_{\Theta}}-\frac{\sigma_{2} \widetilde{m}^{2}}{2 m k_{m}}-\frac{\sigma_{3}\left(\widetilde{D}^{*}\right)^{2}}{2 m k_{D}} \\
& +\frac{\sigma_{1} \Theta^{T} \Theta}{2 k_{\Theta}}+\frac{\sigma_{2} m^{2}}{2 m k_{m}}+\frac{\sigma_{3}\left(D^{*}\right)^{2}}{2 m k_{D}} \\
& +\frac{D^{*}}{m}\left(\left|\xi_{2}\right|-\xi_{2} \tanh \frac{\xi_{2}}{\varepsilon}\right) \\
\leq & -\alpha_{v 1} \xi_{1}^{2}-\alpha_{v 2} \xi_{2}^{2}-\frac{\sigma_{1} \widetilde{\Theta}^{T} \widetilde{\Theta}}{2 k_{\Theta}}-\frac{\sigma_{2} \widetilde{m}^{2}}{2 m k_{m}}-\frac{\sigma_{3}\left(\widetilde{D}^{*}\right)^{2}}{2 m k_{D}} \\
& +\frac{\sigma_{1} \Theta^{T} \Theta}{2 k_{\Theta}}+\frac{\sigma_{2} m^{2}}{2 m k_{m}}+\frac{\sigma_{3}\left(D^{*}\right)^{2}}{2 m k_{D}}+\frac{0.2785 \varepsilon D^{*}}{m} \\
= & -\rho V+\Psi,
\end{aligned}
$$

where

$$
\begin{aligned}
\Psi= & \left(\frac{\sigma_{1}}{2 k_{\Theta}}\right) \Theta^{T} \Theta+\frac{0.2785 \varepsilon D^{*}}{m}+\left(\frac{\sigma_{2}}{2 k_{m}}\right) m \\
& +\left(\frac{\sigma_{3}}{2 m k_{D}}\right)\left(D^{*}\right)^{2}, \\
\rho= & \min \left\{2 \alpha_{v 1}, 2 \alpha_{v 2}, \sigma_{1}, \sigma_{2}, \sigma_{3}\right\} .
\end{aligned}
$$

Solving $V$ from $\dot{V} \leq-\rho V+\Psi$ and from (A.1), one can obtain

$$
0 \leq 0.5 \xi_{1}^{2} \leq V \leq\left(V(0)-\frac{\Psi}{\rho}\right) e^{-\rho t}+\frac{\Psi}{\rho},
$$

where $V(0)=0.5\left(\xi_{1}(0)\right)^{2}+(\widetilde{\Theta}(0))^{T} \widetilde{\Theta}(0) / 2 k_{\Theta}+0.5\left(\xi_{2}(0)\right)^{2}+$ $(\widetilde{m}(0))^{2} / 2 m k_{m}+\left(\widetilde{D}^{*}(0)\right)^{2} / 2 m k_{D}$.

It can be seen that there exists a time $T>0$ such that $\forall t>$ $T,(V(0)-\Psi / \rho) e^{-\rho t} \rightarrow 0$. Then $V$ is bounded by $\Psi / \rho$ for $\forall t>T$. Hence $V$ is uniformly ultimately bounded (UUB); thus the tracking errors $e_{r}=\xi_{1}, \widetilde{D}^{*}, \widetilde{m}, \widetilde{\Theta}$ are also bounded. This further guarantees the boundedness of $\widehat{D}^{*}, \widehat{m}, \widehat{\Theta}$ since $D^{*}, m, \Theta$ are bounded.
From (A.9), the variable $\xi_{1}$ can be expressed as

$$
\left|e_{r}\right|=\left|\xi_{1}\right| \leq \sqrt{2\left(V(0)-\frac{\Psi}{\rho}\right) e^{-\rho t}+\frac{2 \Psi}{\rho}} .
$$

$\forall t>T$, the tracking error $e_{r}=\xi_{1}$ will converge to a compact set $\Omega=\left\{\left|e_{r}\right||| e_{r} \mid \leq 2 \sqrt{\rho / \Psi}\right\}$ since $(V(0)-\Psi / \rho) e^{-\rho t} \rightarrow 0$. Consequently, the control input, $u$, is also bounded.

\section{Conflicts of Interest}

The authors declare that there are no conflicts of interest regarding the publication of this paper.

\section{Acknowledgments}

The authors would like to thank Benedict Lai and Van Than Dung for their help and effort during the wind tunnel tests and also Vigneashwara Pandiyan for piloting the FMW miniUAV during the flight test.

\section{References}

[1] E. C. C. Choi, "Extreme wind characteristics over Singapore An area in the equatorial belt," Journal of Wind Engineering and Industrial Aerodynamics, vol. 83, pp. 61-69, 1999.

[2] W. J. Pisano and D. A. Lawrence, "Autonomous gust insensitive aircraft," in Proceedings of the AIAA Guidance, Navigation and Control Conference and Exhibit, American Institute of Aeronautics and Astronautics, Honolulu, Hawaii, August 2008.

[3] P. Ifju, D. Jenkins, S. Ettinger, Y. Lian, W. Shyy, and M. Waszak, "Flexible-wing-based micro air vehicles," in Proceedings of the 40th AIAA Aerospace Sciences Meeting \& Exhibit, American Institute of Aeronautics and Astronautics, Reno, NV, USA, 2002.

[4] M. Abdulrahim, H. Garcia, and R. Lind, "Flight characteristics of shaping the membrane wing of a micro air vehicle," Journal of Aircraft, vol. 42, no. 1, pp. 131-137, 2005.

[5] H. Garcia, M. Abdulrahim, and R. Lind, "Roll Control for a Micro Air Vehicle Using Active Wing Morphing," in Proceedings of the AIAA Guidance, Navigation, and Control Conference and Exhibit, American Institute of Aeronautics and Astronautics, Austin, Texas, August 2003.

[6] G. Palli and C. Melchiorri, "Model and control of tendonsheath transmission systems," in Proceedings of the 2006 IEEE International Conference on Robotics and Automation, ICRA 2006, pp. 988-993, usa, May 2006.

[7] Y. Lian, W. Shyy, D. Viieru, and B. Zhang, "Membrane wing aerodynamics for micro air vehicles," Progress in Aerospace Sciences, vol. 39, no. 6-7, pp. 425-465, 2003.

[8] Y. Lian, W. Shyy, and R. T. Haftka, "Shape Optimization of a Membrane Wing for Micro Air Vehicles," AIAA Journal, vol. 42, no. 2, pp. 424-426, 2004.

[9] H. Hu, M. Tamai, and J. T. Murphy, "Flexible-membrane airfoils at low reynolds numbers," Journal of Aircraft, vol. 45, no. 5, pp. 1767-1778, 2008.

[10] S. Lee, T. Tjahjowidodo, H. C. Lee, and B. Lai, "Investigation of a robust tendon-sheath mechanism for flexible membrane wing application in mini-UAV,' Mechanical Systems and Signal Processing, vol. 85, pp. 252-266, 2017. 
[11] M. Abdulrahim, H. Garcia, and R. Lind, "Flight Testing a Micro Air Vehicle Using Morphing for Aeroservoelastic Control," in Proceedings of the 45th AIAA/ASME/ASCE/AHS/ASC Structures, Structural Dynamics \& Materials Conference, American Institute of Aeronautics and Astronautics, Palm Springs, Calif, USA, April 2004.

[12] R. De Breuker, M. M. Abdalla, and Z. Gürdal, "A generic morphing wing analysis and design framework," Journal of Intelligent Material Systems and Structures, vol. 22, no. 10, pp. 1025-1039, 2011.

[13] D. P. Raymer, Aircraft Design: A Conceptual Approach And RdsStudent, Software for Aircraft Design, Sizing, and Performance Set (AIAA Education), AIAA, Los Angeles, Calif, USA, 2006.

[14] J. Roskam, Airplane Design: Determination of stability, control and performance characteristics: far and military requirements, DARcorporation, Lawrence, Kansas, USA, 2002.

[15] M. H. Sadraey, Aircraft Design: A Systems Engineering Approach, John Wiley \& Sons, Hoboken, NJ, United States, 2012.

[16] M. Kaneko, T. Yamashita, and K. Tanie, "Basic considerations on transmission characteristics for tendon drive robots," in Proceedings of the Fifth International Conference on Advanced Robotics, 1991.'Robots in Unstructured Environments', 91 ICAR, pp. 827-832, IEEE, Pisa, Italy, June 1991.

[17] J. Song and A. Der Kiureghian, "Generalized Bouc-Wen model for highly asymmetric hysteresis," Journal of Engineering Mechanics, vol. 132, no. 6, pp. 610-618, 2006.

[18] T. N. Do, T. Tjahjowidodo, M. W. S. Lau, and S. J. Phee, "Dynamic Friction-Based Force Feedback for Tendon- Sheath Mechanism in NOTES System," International Journal of Computer and Electrical Engineering, vol. 6, no. 3, pp. 252-258, 2014.

[19] F. Ikhouane, V. Mañosa, and J. Rodellar, "Dynamic properties of the hysteretic Bouc-Wen model," Systems and Control Letters, vol. 56, no. 3, pp. 197-205, 2007.

[20] F. Ikhouane, V. c. Mañosa, and J. Rodellar, "Adaptive control of a hysteretic structural system," Automatica. A Journal of IFAC, the International Federation of Automatic Control, vol. 41, no. 2, pp. 225-231, 2005.

[21] M. Mahvash and A. Okamura, "Friction compensation for enhancing transparency of a teleoperator with compliant transmission," IEEE Transactions on Robotics, vol. 23, no. 6, pp. 12401246, 2007.

[22] H. K. Khalil and J. Grizzle, "Nonlinear systems," vol. 3, Prentice hall Upper Saddle River.

[23] M. Krstic, P. V. Kokotovic, and I. Kanellakopoulos, Nonlinear and adaptive control design, John Wiley \& Sons, Inc., Hoboken, NJ, United States, 1995.

[24] N. S. Khot, F. E. Eastep, and R. M. Kolonay, "Wing twist and camber for the rolling maneuver of a flexible wing without aileron," in Proceedings of the 38th AIAA/ASME/ASCE/AHS/ASC Structures, Structural Dynamics, and Materials, pp. 759-768, American Institute of Aeronautics and Astronautics, Reston, Virginia, April 1997.

[25] D. M. Tang, P. G. A. Cizmas, and E. H. Dowell, "Experiments and analysis for a gust generator in a wind tunnel," Journal of Aircraft, vol. 33, no. 1, pp. 139-148, 1996.

[26] H. Xu, S. Xing, and Z. Ye, "Numerical study of an airfoil/rotatingslotted-cylinder based flutter exciter," Journal of Aircraft, vol. 52, no. 6, pp. 2100-2105, 2015.

[27] Y. Feng, Y.-M. Hu, C. A. Rabbath, and C.-Y. Su, "Robust adaptive control for a class of perturbed strict-feedback nonlinear systems with unknown Prandtl-Ishlinskii hysteresis," International Journal of Control, vol. 81, no. 11, pp. 1699-1708, 2008. 


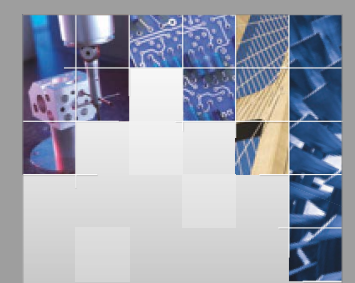

\section{Enfincering}
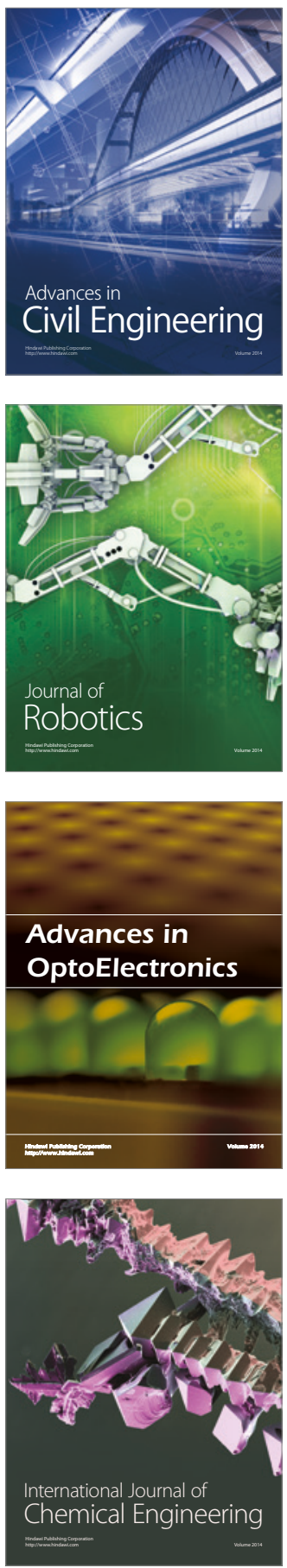

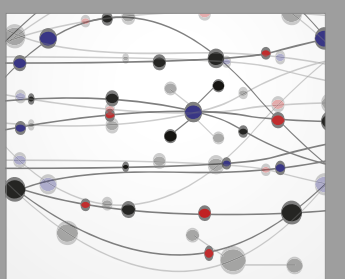

The Scientific World Journal

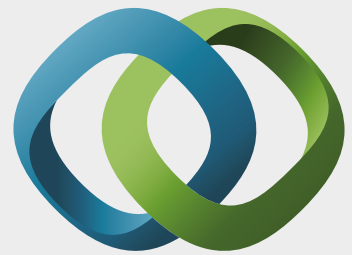

\section{Hindawi}

Submit your manuscripts at

https://www.hindawi.com
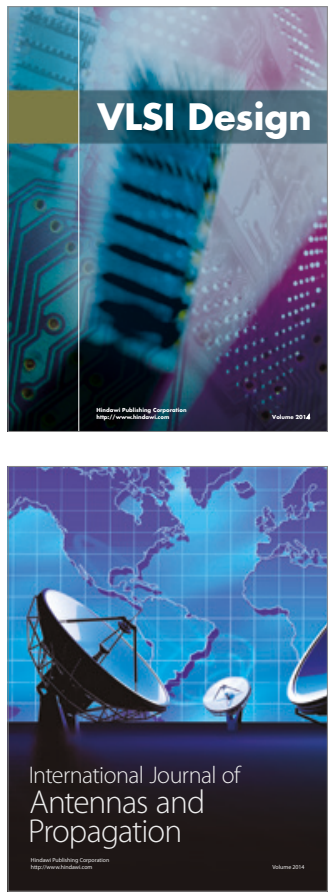

\section{Rotating}

Machinery
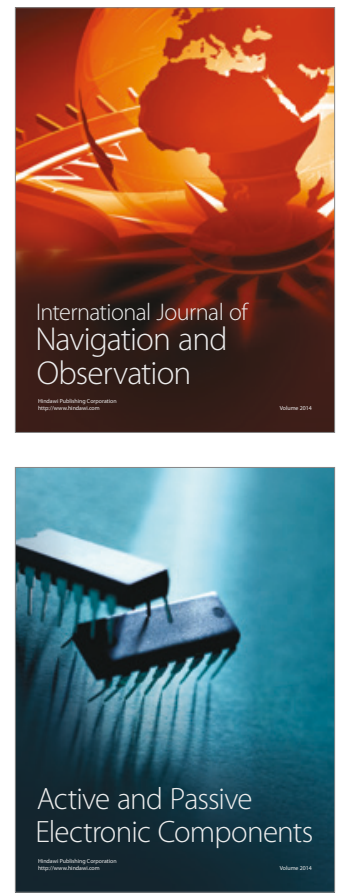
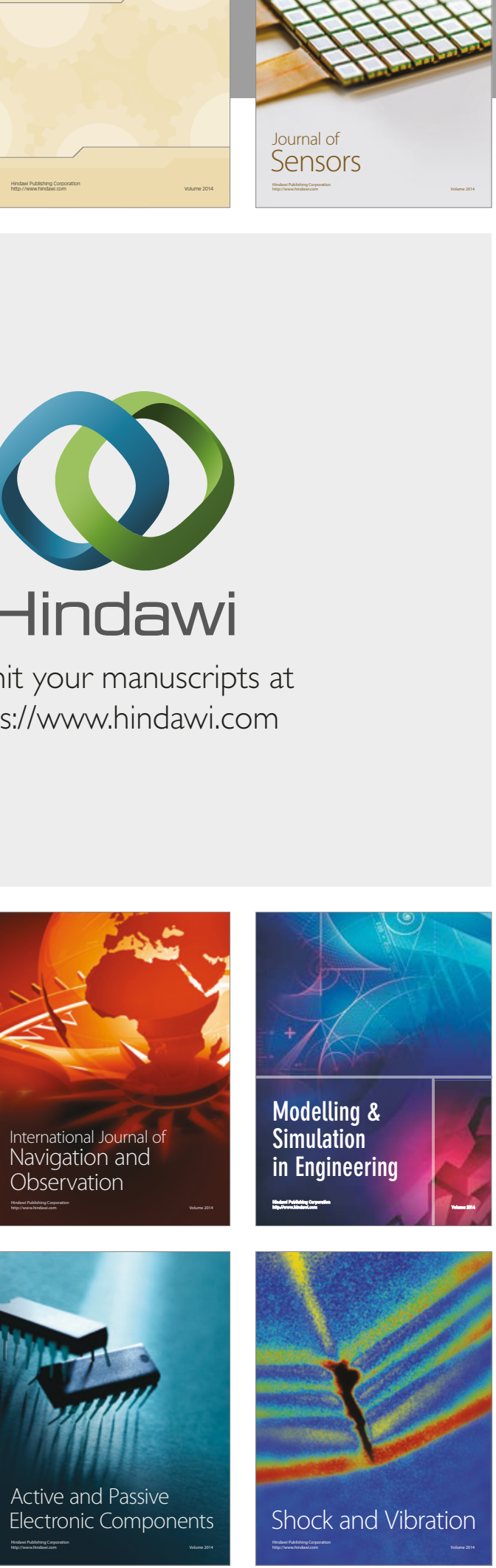
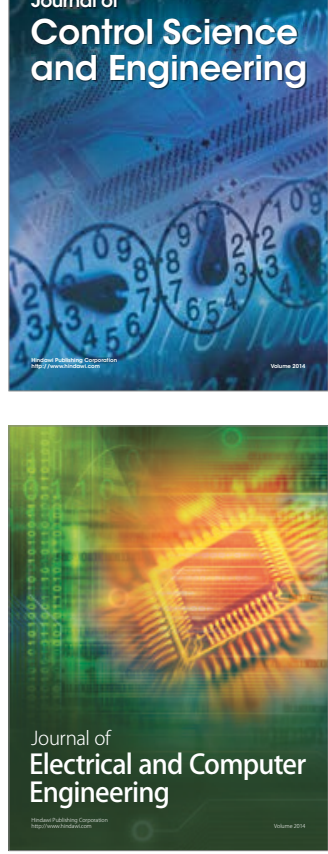

Distributed

Journal of

Control Science

and Engineering
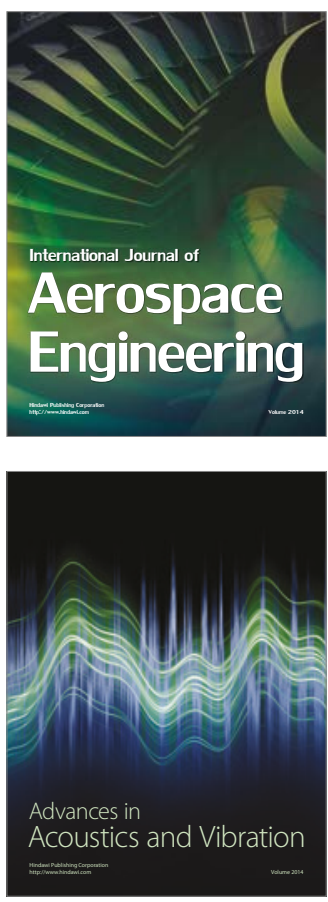

Sensor Networks 